\title{
Modelo de sistema multisensor con enfoquede muestreo multifrecuencia
}

Multisensor system model with multirate sampling approach

\author{
Alfonso Alfonsi ${ }^{1}$ \\ alfalse@yahoo.es \\ Raiza Yánez ${ }^{1}$ \\ ryanezmar@gmail.com \\ Alfonso R. Alfonsi ${ }^{1}$ \\ alf.rafa@gmail.com
}

${ }^{1}$ Grupo de Investigación Arquitecturas de Sistema de Control, Escuela de Ingeniería y Ciencias Aplicadas, Universidad de Oriente, Núcleo de Anzoátegui 
Resumen: En la fase temprana de diseño de un multisensor están las especificaciones funcionales que establecen el comportamiento de sus señales, donde es importante analizar la influencia de los tiempos de muestreo en el despliegue temporal entre los datos de entrada y salida disponibles del multisensor, como del enlace de comunicación que pudieran utilizar. Por tanto, en este trabajo se desarrolla un modelo de sistema multisensor considerando múltiples señales con periodos de muestreo que pueden ser diferentes del proceso y datos de estado, incluyendo la limitación temporal del enlace de comunicación. El sistema consta de los componentes: multisensor y estación base. Además, se desarrolló un modelo de simulación en Scilab/Xcos para ejercitar diferentes escenarios. El modelo ofrece un perfil temporal de la secuencia de datos sensoriales bajo diferentes esquemas de muestreo, permitiendo presentar la evolución de las señales con pérdidas o ausencias de muestras, las cuales al ser tratadas sistemáticamente con operadores multifrecuencia y funciones de interpolación para su reconstrucción, conducen a resultados utilizables para la abstracción del mundo real. Finalmente, la efectividad del modelo se ilustra mediante simulaciones numéricas.

Palabras claves: Esquemas de muestreo, modelo sistemas multifrecuencia, multisensor, simulación.

Abstrac: In the early design phase of a multi-sensor, there are functional specifications that establish the behavior of its signals where it is important to analyze the influence of the sampling rates on the temporal display between the available input and output data from the multi-sensor, such as the communication they could use. Therefore, this work develops a multi-sensor system model considering multiple signals with sampling periods that could be different from the status data and the process itself; including the time-related limitations on the communication link. The system has two components, the multi-sensor and base station. In addition, it was developed a simulation model in Scilab / Xcos for some exercise scenarios. The model offers a time-based profile with the sequence of the data under different sampling schemes allowing to show the evolution of the signals with loss or absence of samples, which when it being systematically treated with multi-frequency operators and interpolation functions for their reconstruction, lead to usable results for real-world abstraction. Finally, numerical simulations illustrate the effectiveness of the model.

Keywords: Multi-sensor, multi-rate systems model, sampling schemes, simulation. 


\section{INTRODUCCIÓN}

Uno de los actores principales producto de las crecientes potencialidades de los sistemas computacionales empotrados asociados a la instrumentación, la comunicación y el control es el multisensor, que según Deb Majumder et al. (2019) incluyen múltiples sensores diferentes, cada uno con sus propias funciones, miden un determinado parámetro de manera independiente y usan algoritmos especiales del procesamiento de señales para combinar todas las mediciones independientes en un valor de medición integrado. Además, ofrece información de estados de operatividad y configuración de parámetros funcionales y facilidades de comunicación. Lo que ha permitido sus progresivas incursiones hacia Redes de Sensores y Actores Inalámbricos (WSAN: Wirelless Sensor and Actors Networks) (Akyildiz y Kasimoglu, 2004; Kakarla et al., 2015), Internet de las Cosas (IoT: Internet of Things) (Muangprathuba et al., 2019; Pradilla et al., 2018) y Sistemas Ciberfísicos (CPS: Cyber-Physical Systems) (Liu et al., 2017; Roehm et al., 2019).

Al respecto, diseñar un multisensor no se escapa de los enfoques principales para el desarrollo de sistemas empotrados, como el codiseño hardware y software, plataforma de diseño y diseño basado en componentes, que tienen en común la descripción del sistema para cumplir especificaciones funcionales de diseño (Ha y Teich2017; Laszlo y Zhexembayeva, 2011; Teich, 2012), que está en consonancia con la norma sobre instrumentos inteligentes ISO/IEC/IEEE 21451 (ISO/IEC/IEEE, 2010a, 2010b).

En este sentido, dentro de las especificaciones funcionales están las secuencias de datos sensoriales provenientes de los múltiples sensores, resultado de las mediciones independientes de las magnitudes del proceso, así como también, los referidos a sus estados y configuraciones, los cuales deben estar disponibles para una red de datos e Internet.

Dichas secuencias están influenciadas por retardos en el tratamiento de las distintas señales que pueden degradar la visión general y completa de las mediciones, al presentarse pérdidas o ausencias de datos sensoriales y perfiles temporales; debido, entre otras razones, a tiempos de muestreo que pudieran ser diferentes con esquemas regulares e irregulares, presencia de medios de comunicación y cálculos computacionales. Por tanto, es fundamental tener herramientas que permitan analizar las relaciones del despliegue temporal junto a las magnitudes disponibles de las entradas y salidas, no solo del multisensor, sino también, del enlace de comunicación; lo cual facilitará una descripción del comportamiento para la toma de decisión, ya sea a nivel de las especificaciones funcionales del diseño, su mantenimiento y actualización.

Si bien es cierto que una de las aristas en la adquisición de las señales de un proceso es la selección de los tiempos de muestreo, dominado formalmente en la literatura del tratamiento digital de señales y sistemas de control digital, donde establecen dependencia a factores como, el ancho de banda, tiempo de respuesta de los sistemas en lazo cerrado, perturbaciones, limitaciones físicas de los sensores y actuadores (Dorf y Bishop, 2017; Lathi y Green, 2019; Oppenheim et al., 2013; Tan y Jiang, 2018), y prevalece la elección convencional de patrones de muestreo regular y uniforme de las distintas variables; no obstante subyace, que la selección del tiempo de muestreo se está discutiendo en varios contextos (Deb Majumder et al., 2019), siendo uno de ellos los sistemas con muestreo no convencional (SMNC) o multifrecuencia (MF).

Desde luego, para el análisis, la modelización y diseño de los sistemas con entradas y salidas que se actualizan a diferentes velocidades, son necesarias otras directrices que permitan al diseñador acomodar múltiples velocidades de datos e implementar los cálculos necesarios requeridos dentro de las capacidades computacionales finitas de un sistema computacional. Como, los muestreadores ascendentes, los muestreadores descendentes, la técnica de elevación y la transformada Z modificada (Bamieh y Pearson, 1991; Cimino y Pagilla, 2010; Coffey y Williams, 1966; Ding et al., 2009; Glasson, 1983; Godbout et al., 1990; Kranc, 1957; Meinsma y Mirkin, 2010a; Salt y Albertos, 2005; Thompson, 1986). 
En consecuencia, este trabajo se centra en el desarrollo de un modelo de sistema multisensor (SMs) desde la perspectiva de los sistemas MF, que considera múltiples señales con periodos de muestreo que pueden ser diferentes, del proceso y datos de estado, incluyendo la influencia temporal del enlace de comunicación, con el fin de ofrecer un perfil temporal de la secuencia de los datos sensoriales, que sirve para comprobar si realmente su comportamiento coincide con las especificaciones deseadas. EI SMs lo forman los módulos: multisensor y estación base. Además, se desarrolla un modelo de simulación en Scilab/Xcos (Scilab, 2020) para el ejercicio de los diferentes escenarios.

Dentro del contexto hay trabajos a lo largo del tiempo que soportan a la MF, desde los enfocados al procesamiento digital de señales haciendo énfasis en la transformación de frecuencias, decimación e interpolación, como las estructuras de Crochiere y Rabiner (1981). Los trabajos de Meinsma y Mirkin (2010a, 2010b) amplían las ideas clásicas del procesamiento digital de señales MF, al revisar el proceso de conversión de frecuencias, como la definición canónica del decimador e identificación del interpolador o expansor correspondiente, definiendo sus propiedades y, muestran que, al tratamiento clásico MF se deben imponer ciertas restricciones matemáticas para establecer sus identidades. Diseñan bancos de filtros polinómicos con menor complejidad. Además, abordan un amplio espectro de problemas de muestreo y reconstrucción no causales, basados en la optimización, traducidos en versiones del teorema de muestreo para la reducción de muestras y reconstrucción a partir de mediciones ruido.

Kordestani et al. (2020) y Yan et al. (2015) presentan estrategias de fusión de datos para estimar el estado de los sistemas multisensores MF, con mediciones faltantes mediantes filtros Kalman MF y algoritmos de fusión recursivo óptimo de datos asincrónicos. Aunque el objetivo de estos trabajos está dirigido a la a fusión multisensor, los modelos presentados son un claro ejemplo de la sinergia entre datos faltantes y MF. También destacan, trabajos que combinan los datos de medición de todos los sensores que utilizan algoritmos especiales para la fusión de datos multisensor (Nwe y Tun, 2017; Ghosal y Rao, 2019).

Salt et al. (2008) introducen lineamientos de los SMNC para ofrecer alternativas referente a los Sistemas de Control Basados en Red (NCS: Networked Control Systems) con el propósito de atender el desfase entre los muestreadores de las partes remota y local, cuando se requiere su sincronización. Acotando, no todos los muestreos podrán ser enviados y ocurrirá una inevitable pérdida de información. Al respecto, infieren aquellos valores de variables que pueden tener una utilidad clara en la aplicación del control, visualizándolos desde la dinámica de la planta y procedimientos basados en señales. Por su parte, Zhu et al. (2016) proponen un modelo de NCS MF con retardos escasos y pérdidas de paquetes, pero esta vez desde un sistema estocástico conmutado de tiempo discreto, garantizando la estabilidad del NCS. Jia et al. (2017) diseñan un controlador basado en un observador para un NCS con muestreo MF para estimar el estado del sistema en tiempo real, producto de una nueva función de Lyapunov-Krasovskii construida para analizar eficientemente el sistema de circuito cerrado en presencia de retrasos inducidos por la red que varían en el tiempo.

Desde los sistemas de control, Albertos y Salt (2011) abordan el problema de controlar las plantas MIMO donde el patrón de mediciones, muestreo y acciones de control no son regulares. Revisan diferentes modelos e informan técnicas de diseño de control basadas en modelos. Zhang et al. (2015) trabajan con sistemas lineales muestreados bifrecuencia en espacio de estados y construyen un controlador conmutado periódico. Aranda-Escolástico et al. (2018) proponen un algoritmo de optimización que suministra, no solo las señales de entrada, sino también sus tiempos de aplicación, y maximiza la tasa de decaimiento del sistema considerando un escenario de doble velocidad con entrada lenta y salida rápida.

En fin, se siguen los lineamientos del Muestreo MF al observar el modelo como una realización discreta considerando un sistema multivariable y MF, donde los periodos de muestreo de las señales $T_{i}$ sean conmensurables, pudiendo ser diferentes, a un periodo global $T_{0}$ y enlace $T_{\text {Enlace }}$. 
El resto del artículo está organizado de la siguiente manera. El modelo del SMs y su estructura formal con enfoque MF se formula en la Sección 2. En la Sección 3, se demuestra un escenario de aplicación mediante la simulación, con un multisensor, estación base y la presencia de un enlace de comunicación, como ejemplo numérico para demostrar la efectividad del modelo. Finalmente, en la Sección 4 se presentan las respectivas conclusiones.

\section{MODELO DEL SISTEMA MULTISENSOR}

El propósito del modelo es representar los recursos disponibles de forma uniforme que permita ofrecer un perfil temporal de secuencias de datos sensoriales, que sirve para comprobar si realmente el comportamiento coincide con las especificaciones deseadas, al tomar en consideración múltiples señales con periodos de muestreo que pueden ser diferentes, del proceso y datos de estado, incluyendo la influencia del enlace de comunicación. En este sentido, se parte de una estructura de un Sistema Multisensor, presentada en la Figura 1, formada por los módulos Multisensor (MS) y Estación Base (EB). A su vez, cada módulo está integrada por componentes operacionales reutilizables.

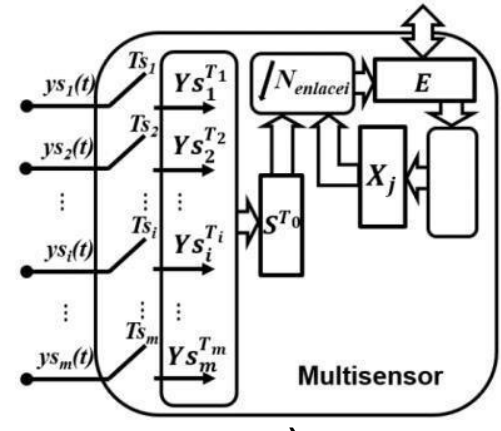

a)

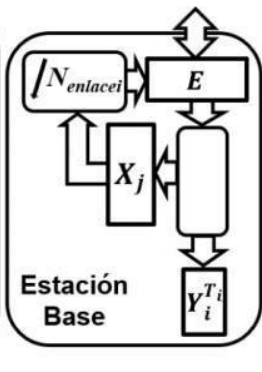

b)

Figura 1. Estructura del Sistema Multisensor basado en Muestreo MF. a) Multisensor. b) Estación Base.

\subsection{Módulo Multisensor}

En primer lugar, se destacan los componentes del módulo MS, indicados en la Figura 1a, formado por los componentes: Manejo de la Medición de Señales Externas, Información y Estado MS y, Preparación para el Enlace de Comunicación. Su función es obtener las señales externas de manera independiente por múltiples sensores diferentes, prepararlos para transformaciones de periodos de muestreo en el SMs y disponerlos en un elemento de datos que se transmitirá a la EB, junto a las respuestas por consultas realizadas desde la EB.

\subsubsection{Componente para el Manejo de la Medición de Señales Externas}

A la izquierda de la Figura 1a, se encuentra el componente que rige la medición de las señales externas de manera independiente por múltiples sensores diferentes, caracterizados por tener enlaces de uso exclusivo para medir magnitudes representadas por las señales continuas $y s_{i}(t)(i=1,2, \cdots, m)$ de las cuales se pueden obtener secuencias de datos tomadas a periodos de muestreo que pueden ser diferentes $T s_{i},\left\{y s_{i}\left(k_{i} T s_{i}\right)\right\}, k_{i} \in Z^{+}$, denotadas por $Y S^{T i}$ (el superíndice indica los tiempos de muestreo). A estas secuencias de datos se le aplica una operación de transformación de periodos de muestreo para obtener el elemento de datos llamado $S^{T s 0}$. Entonces, tomando en consideración el monitoreo de las $Y s_{i}^{T s i}$ se define formalmente dicho componente.

Las secuencias $\left\{y s_{i}\left(k_{i} T s_{i}\right)\right\}$ están relacionadas a valores constantes en los instantes de tiempo $T s_{i}$, siendo su transformada $Z$ expresada como:

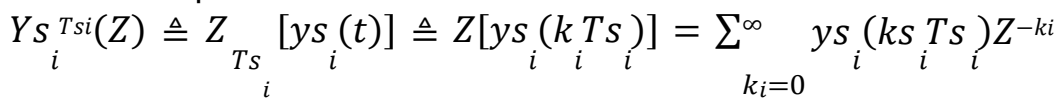


Si los $T s_{i}$ son ampliados a $N s_{i}(i=1,2,3, \cdots, m), N s_{i} \in Z^{+}$, las secuencias de datos tomadas a diferentes periodos de muestreo $N s_{i} T s_{i},\left\{y s_{i}\left(k_{i} N s_{i} T s_{i}\right)\right\}$ denotadas por $Y s_{i}^{N s i T s i}$, tendrán las siguiente transformada Z:

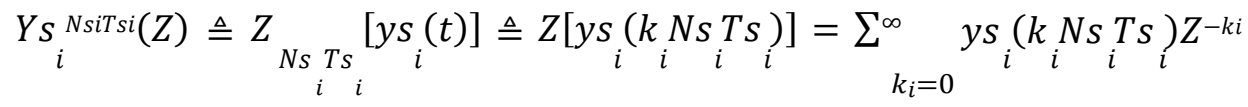

En vista que cada variable tiene su propio periodo de muestreo, el esquema de las secuencias de muestreo está dado por $k_{i}=0,1,2,3, \cdots, N s_{i}-1$. Salt et al. (2014) indican que el significado físico no permite una sustitución trivial. Entonces, una forma de lograr un manejo adecuado a este tipo de sistemas requiere la administración de cada período de muestreo, en el entendido que dichas transformaciones de periodos, entre señales discretas u operaciones de muestreo que involucran bloques de diferente naturaleza, es bastante común en los sistemas MF, lo que lleva a introducir lineamientos en este campo, discutidas entre otras referencias, en Meinsma y Mirkin (2010a) y Oppenheim et al. (2013).

Para tal fin, se define el periodo de muestreo global o hiperperiodo $T s_{0}$ como el intervalo de repetición del esquema de muestreo, el cual se calcula como el mínimo común múltiplo de los periodos de muestreo en el sistema. La relación entre $T s_{0}$ y $T s_{i}$ es el número de muestras $N s_{i}$ de $y s_{i}(t)$ en $T s_{0}$ dado por:

$$
N s_{i}=\frac{T s_{0}}{T s_{i}}
$$

En MF es importante especificar el $T s_{0}$ porque que ayuda reunir a un conjunto de muestreos de las señales en este intervalo. Lo que permite tener el esquema de frecuencia de muestreo $k_{i}$. En la Figura 2 se presentan las muestras $k_{i},\left(0 \leq k_{i} \leq N s_{i}\right)$ tomadas de cada sensor $i$ a $T s_{i}$.

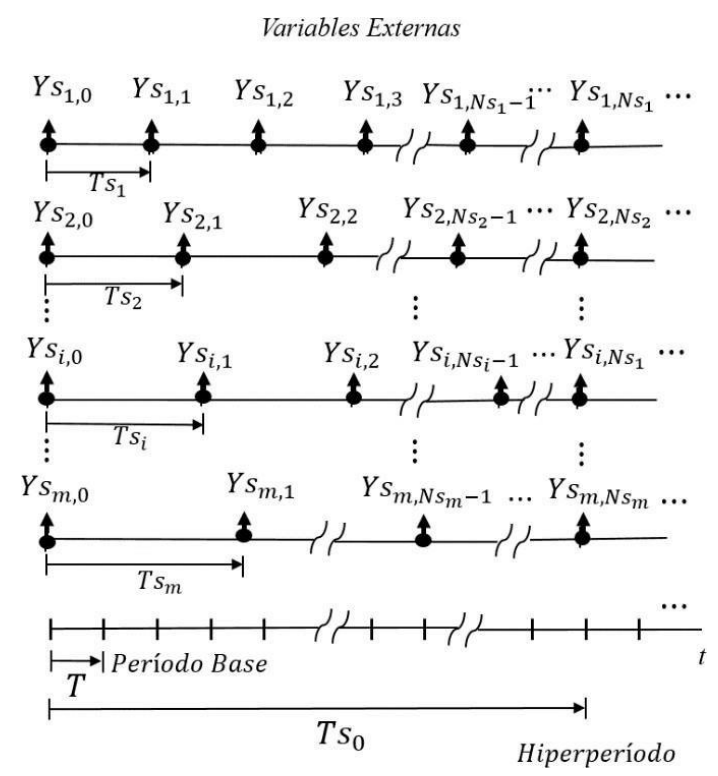

Figura 2. Esquema de muestreo MF regular de las variables externas preparadas a $T_{s 0}$.

Entonces, ordenando los elementos $Y S^{T S i}$ se define un conjunto en el dominio temporal como se muestra en (4), con una dimensión de $\left(m x \sum_{i=1}^{m} N_{i}^{i} S_{i}\right)$ por cada hiperperiodo $T s_{0}$ considerado. 


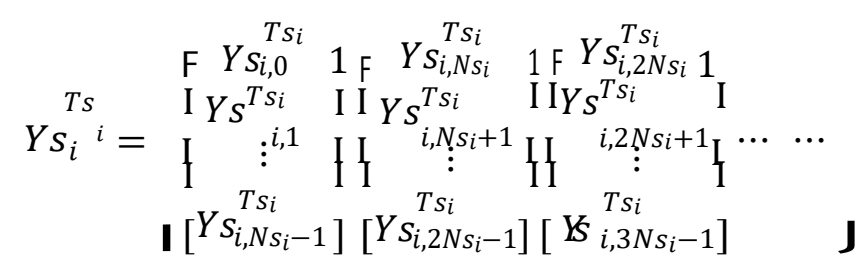

Para sincronizar las muestras $Y s_{i}^{T s i}$ se deben transformar los periodos de muestro a $T s_{0}$. Entonces, tomando la transformada $Z$ referida al período $N s_{i} T s_{i}$, como en (3), es posible expresar formalmente, la operación de reducción de frecuencia en dichas señales discretas con el operador de Salto $(S k p)$, denotado por Skpo $\downarrow N$, para una secuencia digital actuando de la siguiente forma:

$$
S k p:\left[Y^{T}(Z)\right]^{N T} \triangleq \mathfrak{y}^{\top T}\left(Z^{N}\right) \triangleq \sum_{k=0}^{\infty} y(k N T) Z^{-k N}=Y^{N T}\left(Z_{N}\right)
$$

Es decir, crea una $N s_{i} T s_{i}$-secuencia a partir de una $T s_{i}$-secuencia, donde la secuencia de salida es igual a la primera señal de cada grupo de $N s_{i}$ muestras consecutivas de la señal de entrada, eliminando las $N_{i}-1$ muestras de este grupo. Se comporta como un reductor de frecuencia. Desde el contexto, los argumentos $k$ (de la secuencia) y $Z$ (en el dominio de la transformación) serán omitidos, de aquí en adelante, para simplificar la manipulación de las operaciones. Para más detalles del operador $S k p$ consulte las referencias MF indicadas en este trabajo, siendo algunas de ellas Meinsma y Mirkin (2010a), Oppenheim et al. (2013), Salt J. y Albertos P. (2005) y Salt et al. (2014).

Por tanto, al aplicar el operador $S k p$ da como resultado un conjunto en el dominio temporal como se muestra en (6):

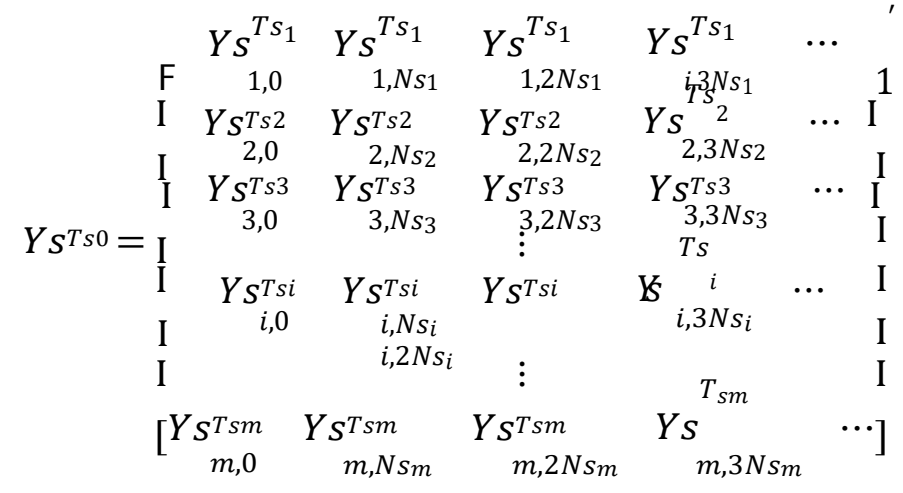

En este sentido, el operador $S k p$ se emplea para representar matemáticamente tal reducción y es la forma más natural de hacerlo. Esto permite eliminar de forma intencionada muestras y, así en este caso se sabe con certeza qué muestras son usadas al hacer la reducción y qué muestras no lo hacen, minimizando la incertidumbre que ocasiona no conocer las muestras no tomadas en cuenta.

Lo anterior permite definir el elemento de datos $S^{T 0}$ del componente de medición de las señales externas, conformado por $Y s^{T s 0}\left(N s_{i} T s_{i}\right.$-secuencia de $\left.Y S_{i}^{T s i}\right)$.

\subsubsection{Componente de Información y Estado MS.}

Este componente hace lo necesario para representar las señales discretas $X_{j}, j \in Z^{+},(j=1,2, \cdots, p)$ que contienen la información interna de los estados de operación del multisensor, posiblemente para fines de diagnóstico, configuración o consulta por parte de la EB. Es un componente común en la estructura del SMs. Se pueden manipular haciendo las transformaciones MF digitales debidas y sustituciones de parámetros para caracterizarlas. Es importante señalar, que pudiera obtener la configuración de algún parámetro funcional o control local eminentemente digital, para aplicaciones de CPS. No obstante, en este trabajo solo se argumentará generalmente la señal discreta y su tratamiento 
MF. CPS. No obstante, en este trabajo solo se argumentará generalmente la señal discreta y su tratamiento MF.

\subsubsection{Componente de Preparación para el Enlace de Comunicación.}

El módulo necesita disponer de un enlace de comunicación que se activa a $T s_{\text {enlace }}$ y proporciona la capacidad de transformación a los períodos de muestreo, como se formula en (7).

$$
T s_{\text {enlace }}=N s_{\text {enlacei }} T s_{i}, N s_{\text {enlacei }} \in Z^{+}, T s_{\text {enlace }} \geq T s_{0}
$$

La caja $\downarrow N_{\text {enlacei }}$, Figura 1a, representa la operación del aumento del periodo de muestreo o $S k p$ que prepara a $Y s^{T s 0}$ a $T s_{\text {enlace. }}$. Es decir, crea una $N s_{\text {enlacei }} T s_{i}$-secuencia a partir de una $T s_{i}$-secuencia, donde la secuencia de salida es igual a la primera señal de cada grupo de $N s_{\text {enlacei }}$ muestras consecutivas de la señal de entrada, eliminando las $N_{\text {enlacei }}-1$ muestras de este grupo. Similar al caso de $Y S^{T s 0}$, se comporta como un reductor de frecuencia, minimizando la incertidumbre que ocasiona no conocer las muestras que se descartan.

Si se considera $B$ como el número de bytes que representa a un elemento de $Y s^{T S}$ y $Y^{T s e n l a c e}$, entonces se puede calcular la longitud $P Y s$ en bytes de $Y^{T s}$ cada $T s_{0}$ con (8); y con (9) a $P Y_{\text {enlace }}$ cada $T s_{\text {enlace }}$ de $Y^{T} s_{\text {enlace }}$ :

$$
\begin{gathered}
P Y s=B m \sum_{i=1}^{m} N s \\
P Y s_{\text {enlace }}=B m
\end{gathered}
$$

Por tanto, al aplicar el operador $S k p$ y tomando $T s_{\text {enlace }}=T s_{0}$ da como resultado:

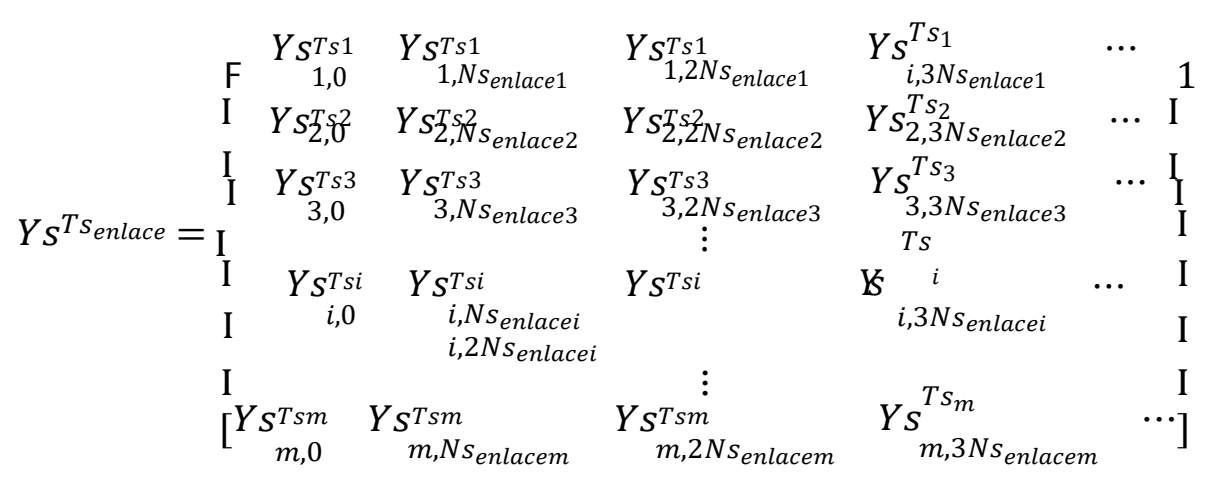

Si $T s_{\text {enlace }}>T s_{0}$, entones $Y s^{T s e n l a c e}$ solo tendría las muestras debidas a $N s_{\text {enlacei }}$ como está establecido.

Ahora bien, con $Y s^{T s e n l a c e}$ y $X_{j}$ se define el elemento de datos $E_{z}, z \in Z^{+},(z=1,2, \cdots, n)$ que es uniforme para las comunicaciones del SMs, siendo este:

$$
E=\left\{\left(Y S^{T \text { senlace }}\right),(X)\right\}
$$

\subsubsection{Variables Externas del MS para uso del Enlace.}

En la Figura 3 se muestra la estructura MF para los esquemas de muestreo que facilita el análisis, imponiendo esquemas de muestreo regular con características de sincronización (secuencias que se repite cada $T s_{0}$ ), considerando un enlace de comunicación transmitiendo a $T s_{\text {enlace }}$. Se toman las señales provenientes de la medición del proceso o entorno que generan las señales discretas $Y s_{i}^{T s i}$ a periodos de muestreos $T s_{i}$, de los cuales se generan los $Y s^{T s 0}$. Posteriormente, son sometidas a una 
conversión digital-digital implementando la operación de $S k p$ para $E_{z}$ que adapta las exigencias del enlace a periodo $T s_{\text {enlace }}$, formando a $Y S^{T s e n l a c e}$.

El muestreo general $T s_{0}$ agrupa a los $T s_{i}$ y, el $T s_{\text {enlace }}$ cumple con las condiciones explícitas por los periodos de muestreo en el enlace de comunicación. En el entendido que la longitud $E_{z}$ está definida y se puede calcular por PYs $s_{\text {enlace }}$ de (9).

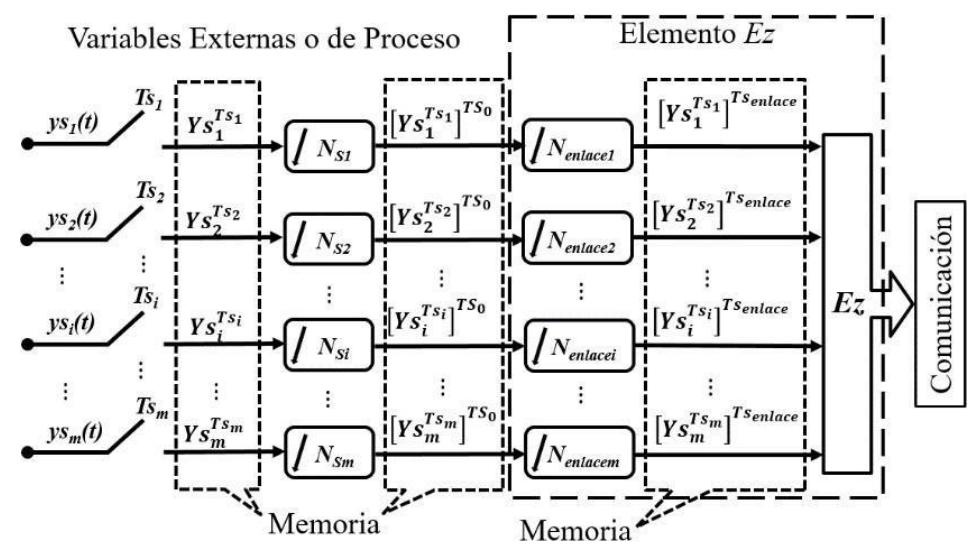

Figura 3. Estructura MF de las señales del MS para la transmisión de variables externas.

Al preparar $Y s^{T s e n l a c e}$ y $E_{z}$ se pueden presentar dos casos, que se consideran ideales, con $T s_{\text {enlace }} \geq$ $T s_{0}$. Uno, cuando los $T s_{i}$ son constantes e iguales y, otro con los $T s_{i}$ constantes y diferentes.

Cuando los $T s_{i}$ son iguales y $T s_{\text {enlace }}=T s_{0}$, el número de muestras $N s_{i}$ de cada señal en $T s_{0}$ estarán disponibles para su comunicación. Sin embargo, cuando $T s_{\text {enlace }}>T s_{0}$ solo las muestras involucradas para cada señal $i$ en el nuevo $T S_{\text {enlace }}$ lo estarán. Lógicamente, existirá una inevitable pérdida de datos. El número de muestras descartadas para la transmisión se incrementará, a medida que $T S_{\text {enlace }}$ aumenta. Es importante señalar que se cumple que $T s_{\text {enlace }}=N s_{0 \text { enlace }} T s_{0}$.

Por otro lado, si los $T s_{i}$ son diferentes, también lo son el número de muestras $N s_{i}$ de cada señal en $T s_{0}$, es decir el número $Y s_{i, k i}^{T S i}$ serán variables, y algunas no consideradas en la transmisión con $T s_{\text {enlace }} \geq$ $T s_{0}$. Habrá una inevitable pérdida de datos y el número aumenta, a medida que $T s_{\text {enlace }}$ aumente, aun cuando fueron medidos, tratados y almacenados.

En la Figura 4 se ilustra el comportamiento de una señal $y s_{i}(t)$ cuando de ejecuta $S k p$ tanto para el $T s_{0}$ como para el enlace con $T s_{\text {enlace }} \geq T s_{0}$, lo que ayuda a visualizar lo descrito anteriormente. 


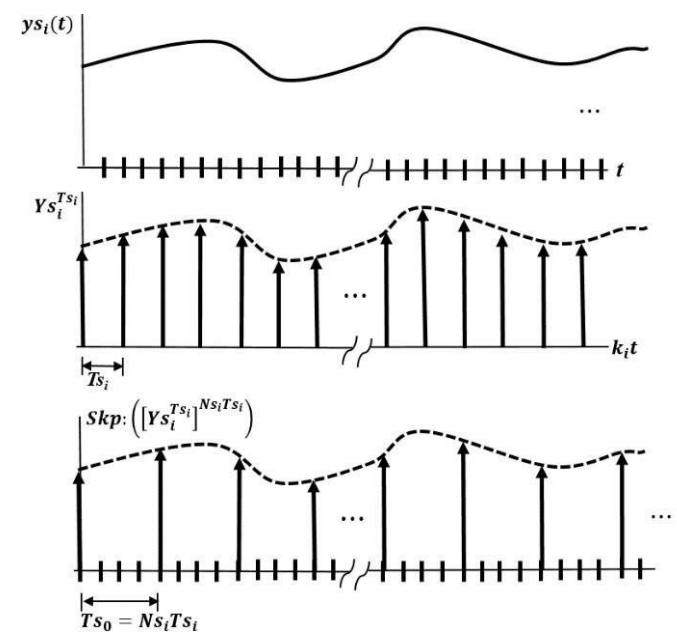

(a)

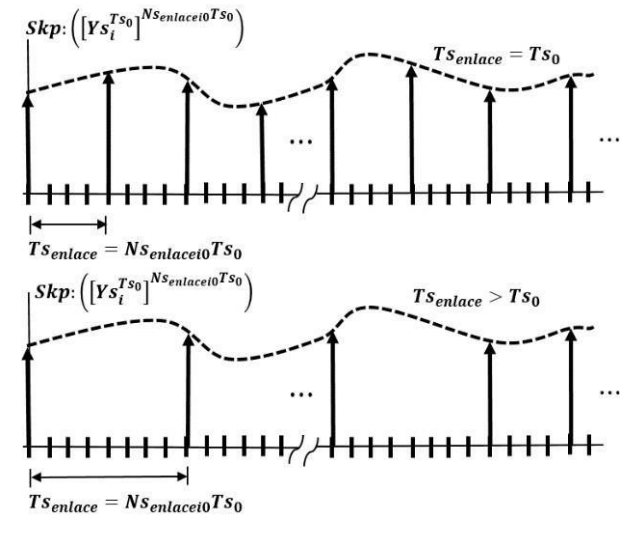

(b)

Figura 4. Comportamiento de $y s_{i}(t)$ con el operador Skp. (a) Al obtener $Y s^{T S 0}$. (b) Al obtener $Y s^{T s e n l a c e}$.

En este punto merece destacarse la consideración de retardos al preparar $E_{z}$ antes de que el enlace recoja el último y lo transmita. En esta materia, haciendo una generalización, se puede modelar considerando $T s_{\text {enlace }}>T s_{0}$, argumentando que $E_{z}$ debe estar disponible para el enlace, por tanto al introducir el retardo $\Delta s_{\text {enlace }}$ recogería tal consideración, mostrada en la Figura 5 . No obstante, en la subsección 3.4 se retoma este aspecto en el ejemplo numérico. También, se conserva la sincronización entre los tiempos de muestreo involucrados.

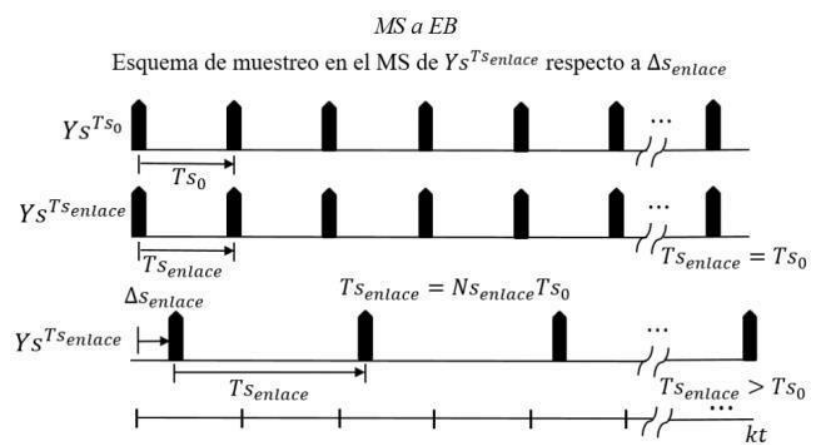

Figura 5. Evolución del muestreo en el MS de $E_{z}=Y S^{T s e n l a c e}$ respecto a $\Delta S_{\text {enlace }}$.

\subsection{Módulo Estación Base}

En este punto se destacan los componentes del módulo EB, indicados en la Figura 1b, donde su insumo principal es el elemento de datos $E_{z}$ indicado en (11). Su función es proveer información para el funcionamiento del SMs, siendo la interfaz entre el usuario y el sistema. Además, es receptor de datos, tanto sensoriales como de respuestas a las consultas, provenientes de los MS. Lo conforman los componentes: Preparación para el Enlace de Comunicación EB, Información y Estado EB y, Procesamiento de Datos Sensoriales del SMs.

\subsubsection{Componentes de Preparación para el Enlace de Comunicación e Información y Estado EB.}

En la parte superior central de la Figura 1b están dos componentes; uno, el de Preparación para el Enlace de Comunicación EB y otro de, Información y Estado EB, las cuales se explican a continuación. 
Primero, la EB recibe al elemento de datos $E_{z}$, alojándolo en el Componente de Preparación para el Enlace de Comunicación con extensión EB en modo recepción a $T S_{\text {enlace, }}$, discriminando sus elementos $X_{j}$ y $Y S^{T s e n l a c e}$, respectivamente.

Las $X_{j}$ que llegan a la EB son asistidas por el Componente de Información y Estado, esta vez con extensión a la EB; también en este componente, es donde convergen y se atienden a otros datos internos de estado de la EB, producto de las funciones particulares de este. Es importante señalar, que estas $X_{j}$ pudieran obtenerse por la configuración de algún parámetro funcional o control local, eminentemente digital enmarcadas en aplicaciones de CPS. No obstante, en este trabajo solo se argumenta de forma general las señales discretas y sus tratamientos MF a consultas realizadas a los MS.

Por otro lado, los datos sensoriales producto de la medición de las señales externas en cada MS $\left(Y S^{T s e n l a c e}\right)$ son enviadas al Componente de Datos Sensoriales para su procesamiento y utilización, el cual se define posteriormente.

Si alguna nueva petición por parte de la EB sucediera, se conformaría nuevamente el elemento $X_{j}$, que sería entregado al componente de Preparación para el Enlace de Comunicación en modo transmisión, con la diferencia que $E_{z}$ estaría formada por $X_{j}$. La caja $\downarrow N_{\text {enlace }}$, de la Figura $1 \mathrm{~b}$, representa la operación de aumento del periodo de muestreo o $S k p$ la cual prepara a $X_{j}$ a $T s_{\text {enlace, }}$, siendo una conversión digital-digital para conservar el estilo MF.

Como se mencionó anteriormente, estos componentes son comunes en la estructura del SMs, siendo utilizados por el sistema según sus funciones de MS o EB, respectivamente. Entonces, se pueden manipular haciendo las transformaciones MF digitales debidas y sustituciones de parámetros para caracterizarlas. Es decir que las operaciones MF se aplican en toda su extensión a las variables y conversiones digital a digital.

\subsubsection{Componente de Procesamiento de Datos Sensoriales del SMs}

Al ubicarse en la parte inferior derecha de la Figura $1 \mathrm{~b}$, está el componente de procesamiento de datos sensoriales provenientes de los Ms. Su objetivo va más allá de las secuencias ordenadas del mundo digital, con ellas se toman las decisiones del sistema. Para ello, se debe procesar al conjunto de muestras digitales con el fin de tomar conocimiento de las señales que se obtuvieron, y de manera natural representar el sistema físico, haciendo el tratamiento de datos, extracción de información, así como también, concentrar la información restante en valores que pueden ser usados como centro de entrada de los respectivos modelos.

De (11) se toma a $Y S^{T s e n l a c e}$, siendo consecuencia de haber aplicado el operador $S k p$ debido a que $T s_{\text {enlace }} \geq T s_{0}$.

En la EB se debe disminuir la frecuencia a las señales, en este caso con el operador de Exp, definido a continuación:

$$
\begin{aligned}
& \text { Exp: }\left[Y_{S^{N s e n l a c e}}^{T s_{i}}\left(Z_{N s_{\text {enlace }}}\right)\right]^{T s_{i}} \triangleq \hat{Y}^{T s_{i}}\left(Z^{\text {Nsenlace }}\right) \triangleq \\
& \triangleq \sum_{k_{i}=0}^{\infty} \hat{\exists}\left(k T_{i} s\right) Z^{-k_{i} N s_{\text {enlace }}} \begin{array}{c}
\hat{\mathbf{y}}\left(k_{i} T s_{i}\right)=y s\left(k_{i} T s_{i}\right) ; \forall k_{i}=\lambda N s_{\text {enlace }} \\
\hat{y}\left(k_{q} T c u_{q}\right)=0 ; \forall k_{q} \neq \lambda N c u_{q}
\end{array} \\
& {\left[y s^{N s_{\text {enlace }} S_{i}}\right]^{T s_{i}}[k]=\left\{\begin{array}{c}
y s_{i}^{N s e n l a c e}{ }^{T s_{i}}\left[k_{i} / N s_{\text {enlace }}\right], \\
0,
\end{array}\right.}
\end{aligned}
$$

Es decir, se crea una $T_{i}$-secuencia a partir de una $N_{\text {ienlace }} T_{i}$-secuencia, donde la secuencia de salida es igual secuencia de entrada cada $N_{\text {ienlace }}$ muestreos (toma de la primera muestra), fijando en cero (0) los 
$N_{\text {ienlace }}-1$ muestreos intermedios. Se reduce el periodo de muestreo por un factor $N_{\text {ienlace }}$, por tanto se incrementa la frecuencia por el mismo acto.

Entonces, como $Y S^{\text {NsenlaceTs }}$ i debe incrementar las frecuencias de muestreo se utiliza el operador Exp definiendo las $Y S E^{T S i}$ en (13) con una dimensión ( $m x \sum_{i=1}^{m} N s \underset{\text { enlacei }}{\text { ) , por cada Ts }}$ enlace

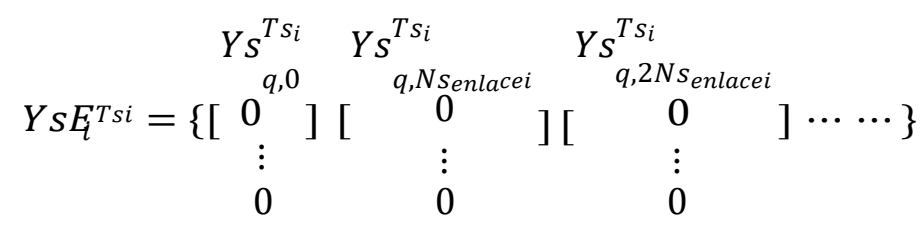

Siguiendo con los operadores MF, también se usa el operador de $R p t$ que realiza la conversión de frecuencia mediante la réplica de muestras. Aquí se crea una $T s_{i}$-secuencia a partir de una $N s_{e n l a c e i} T s_{i^{-}}$ secuencia, replicando los $\mathrm{Ns}_{\text {enlacei- }}-1$ muestreos intermedios, con lo cual resulta evidente que realiza un incremento de frecuencia.

$$
R p t:\left[Y S^{N \text { senlacei } T s_{i}}\left(Z_{\text {Ns }} \text { enlacei }\right)\right]^{T s_{i}}
$$

Hay una relación directa entre los operadores $R p t$ y Exp. El operador $R p t$ es el resultado de una operación de Exp para incrementar la frecuencia seguida de una operación de retención para replicar $N s_{\text {enlacei }}-1$ veces la primera de cada grupo de $N_{\text {enlacei }}$ muestras.

Entonces, como $Y S^{N s e n l a c e i T s} s_{i}$ deben incrementar las frecuencias de muestreo se utiliza el operador $R p t$ definiendo las $Y S R^{T s i}$ en (15) con una dimensión $(m x \sum_{i=1}^{m} N S \underbrace{}_{\text {enlacei }})$, por cada hiperperiodo o Ts enlace

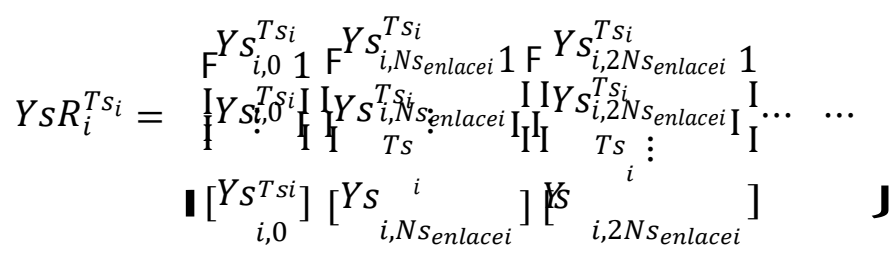

Para más detalles del operador Exp y Rpt consulte las referencias MF indicadas en este trabajo, siendo algunas de ellas Meinsma y Mirkin (2010a), Oppenheim et al. (2013), Salt J. y Albertos P. (2005) y Salt et al. (2014).

Así mismo, se pueden utilizar algoritmos para recuperar o estimar datos, dados una serie parcial de ellos. Uno de éstos es la interpolación mediante trazadores (splines) que estima valores intermedios entre datos definidos y está documentado en varios textos (Chapra y Canale, 2015; Goyal y Dhingra, 2019; Lathi y Green, 2019). Aquí, no se pretende un estudio exhaustivo de dicha interpolación, solo presentarla como ejemplo a seguir. En este trabajo los puntos considerados son los datos obtenidos de $Y^{\text {Tenlace }}$ que después de ejecutar el algoritmo se obtendrá el $Y I_{i, k_{i}}^{T i}$, que representa las señales externas del SMs procesadas.

\subsubsection{Variables Externas del MS en la EB.}

Cuando $E_{Z}$ llega a la EB, la $Y s^{T \text { senlace }}$ debe ser tratada según los objetivos que se persigan, siendo algunos, el monitoreo y observación de las variables para la toma de decisiones, cerrar lazos de sistemas de control remoto, ser usados para CPS, etc. Por tanto, es imprescindible hacer el procesamiento de las señales necesarias para observar los periodos de muestreo de origen. Aquí se plantean dos casos de interés científico y práctico respecto a lo que sucede con las muestras transmitidas desde MS y sus procesamientos, en el receptor o EB. Una dirigida por las restricciones que imponen las aplicaciones que han sido diseñadas a periodo de muestreo de origen. Otra, cuando se monitorean señales para obtener 
características que pueden ser utilizadas en la obtención de modelos, y debido a los efectos del enlace de comunicación se presentan ausencias de algunas muestras.

Para el primer caso, la señal $Y S^{T s e n l a c e}$ debe someterse a una transformación de período de muestreo, observando que $T s_{\text {enlace }} \geq T s_{0}$, donde se requiere muestras a instantes $T s_{i}$. Esto sucede cuando del lado del receptor pudiera estar un controlador discreto remoto, que es diseñado a $T s_{i}$. O, se necesita sincronizar algoritmos que dependen del tiempo de muestreo origen. En este caso, se utilizan los operadores MF Exp y Rpt dependiendo del objetivo que se persiga. Se tiene en cuenta que $T s_{\text {enlace }}=$ $N s_{\text {enlacei }} T s_{i}$.

En la Figura 6 se muestran los casos aplicando lo anteriormente descrito, $Y s E^{T s i}$ y $Y s R^{T s i}$, en sus literales a y b, cuando $T s_{i}$ son constantes e iguales y constantes y diferentes, respectivamente.

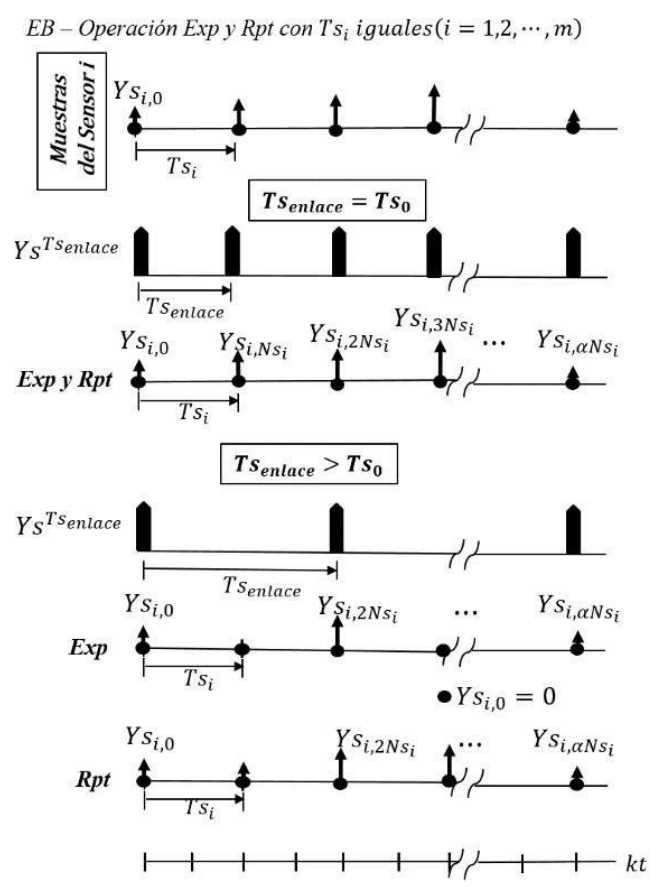

(a)

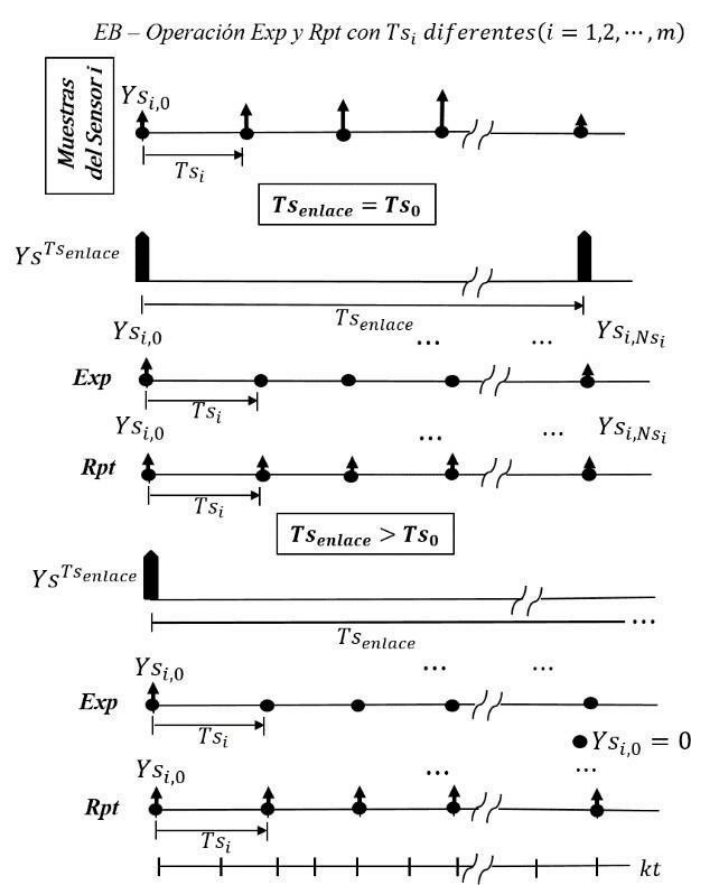

(b)

Figura 6. Evolución del muestreo en la EB aplicando Exp y Rpt con $T s_{\text {enlace }} \geq T s_{0}$. (a) $T s_{i}$ son iguales. (b) $T s_{i}$ diferentes.

En la Figura 7 se presenta el comportamiento de la señal $Y s^{T s e n l a c e}$ una vez que se ejecuta la operación MF $\operatorname{Exp}\left(Y s E^{T s i}\right)$ y $R p t\left(Y s R^{T s i}\right)$, así como también la ilustración al procesar los datos interpolando mediante trazadores cúbicos $\left(Y S I^{T s i}\right)$. 


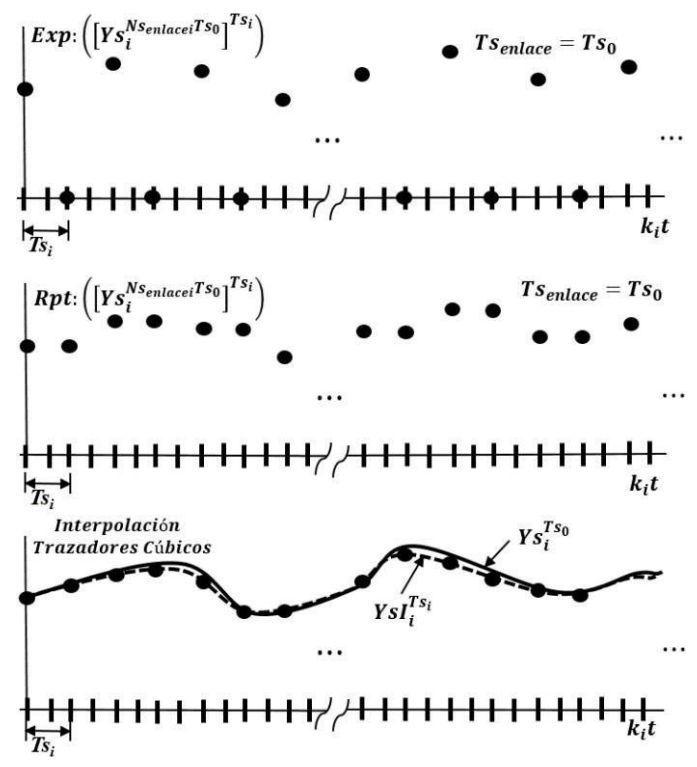

Figura 7. Comportamiento de $y s_{i}(t)$ bajo $E x p, R p t$ e Interpolación mediante trazadores cúbicos.

\section{RESULTADOS}

En esta sección se presenta un escenario de aplicación mediante la simulación como ejemplo numérico para demostrar la efectividad del modelo SMs MF, con un MS y EB en presencia de un enlace de comunicación.

En el SMs se identifican el MS y una EB que tienen un enlace de comunicación. Ambienta sensores cercanos de exteriores en aplicaciones de Agricultura de Precisión. El MS mide señales externas de Temperatura del Aire (TA), Humedad Relativa del Aire (HR) y Temperatura del Suelo (TS). Las variables internas de consulta son Nivel de Carga de la Batería (Bat) (condición inicial 20\%) y Memoria Utilizada (MU) (condición inicial: 10\% MS y 50\% EB) del MS y EB, respectivamente. La simulación de Bat y MU no se presenta en detalle, debido a que sus valores están disponibles en el tiempo, además el procedimiento del tratamiento de las señales son discretas, siendo sus tiempos de entrega son $T d_{r}=\left[\begin{array}{ll}20 & 20\end{array}\right] \min$.

Por tanto se hace énfasis en las señales externas del MS. Los modelos utilizados son de primer orden que se manifiestan en la literatura de los Sistemas de Control (Dorf y Bishop, 2017) y sus parámetros han sido calculados usando datos suministrados de diferentes referencias, entre las cuales se encuentran, Muangprathuba et al. (2019) y Sabri et al. (2012).

Se utiliza el software de simulación de análisis numérico computacional de código abierto Scilab (Scilab, 2020) soportado por Engineering System International Group (https://www.scilab.org/) para la solución de problemas en los ámbitos de los sistemas de control, el procesamiento de señales y otras aplicaciones matemáticas.

El objetivo que se persigue es simular el SMs con las características indicadas anteriormente, siguiendo los casos que a continuación se indican:

1. Esquemas de muestreo convencional considerando el enlace de comunicación a $T s_{\text {enlace }}$ arbitrario, los $T s_{i}$ pueden ser constantes y diferentes.

2. Esquemas de muestreo regular con características de sincronización (secuencias que se repite cada $\left.T s_{0}\right)$, considerando el enlace de comunicación a $T s_{\text {enlace }} \geq T s_{0}$, con $T s_{i}(i=1,2,3)$ son constantes, iguales. 
3. Esquemas de muestreo regular con características de sincronización (secuencias que se repite cada $\left.T s_{0}\right)$, considerando el enlace de comunicación a $T s_{\text {enlace }} \geq T s_{0}$, con $T s_{i}(i=1,2,3)$ son constantes, diferentes.

4. Esquemas de Muestreo con $T s_{i}$ constantes y diferentes con retardos en espera de la activación del enlace a $T$ senlace $_{\text {. }}$

\subsection{Caso Uno: Esquemas de Muestreo Convencional}

Los datos ingresados para la simulación son $T s_{i}=\left[\begin{array}{lll}20 & 20 & 20\end{array}\right]$ min y $T s_{\text {enlace }}=60$ min y 120 min, respectivos a los escenarios planteados.

En la Figura 8a están las salidas de las variables externas TA, HR y TS tomadas de los respectivos sensores del MS con los $T s_{i}$ indicados y se despliegan sin contratiempos, durante 24 horas. Por su parte la Figura $8 \mathrm{~b}$ se hace una simulación variando $T s_{\text {enlace }}=6$ unidades $(60 \mathrm{~min})$ para las primeras doce horas y 12 unidades (120 min) las siguientes horas. Con lo que se corrobora la notoria pérdida de datos en el momento de la transmisión al utilizar el enlace de comunicación, la cual es aleatoria porque depende del $T$ senlace, $_{\text {lo }}$ que podría traer consecuencias del lado del receptor (EBP) al necesitar los datos o una buena aproximación de los comportamientos de los procesos, que están ocurriendo en el MS para la toma de decisiones. Pudiendo mencionar, inconvenientes al establecer controladores remotos, por el asunto de sus diseños, donde se ejecuten algoritmos para recuperar datos ausentes y sistemas que aplican fusión e integración de sensores, entre otros.

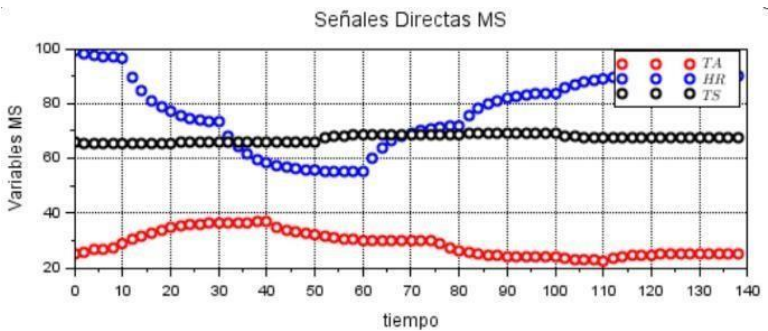

(a)

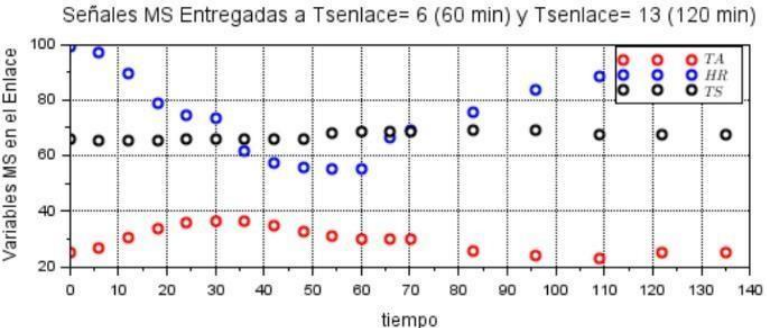

(b)

Figura 8. Salidas de las variables externas del MS TA, HR y TS del MS muestreo convencional. a) Directas del MS. b) Entregadas al Enlace con $T s_{\text {enlace }}=60$ min y $120 \mathrm{~min}$.

\subsection{Caso Dos: Esquemas de Muestreo con $T s_{i}$ constantes, iguales y $T s_{\text {enlace }} \geq T s_{0}$.}

En las dos subsecciones siguientes se presentan los resultados de la simulación cuando $T s_{i}$ son constantes e iguales, con $T s_{\text {enlace }}=T s_{0}$ y $T s_{\text {enlace }}>T s_{0}$, respectivamente. Se sigue las visualizaciones con las salidas de las variables internas y externas.

\subsubsection{Esquemas de Muestreo con $T s_{i}$ constantes, iguales y $T s_{\text {enlace }}=T s_{0}$.}

Los datos a ingresar para la simulación son $T s_{i}=\left[\begin{array}{lll}20 & 20 & 20\end{array}\right]$ min, y $T s_{\text {enlace }}=2$ o 20 min. Después de la simulación se generan las señales para 24 horas.

La Figura 9a representa el comportamiento de las señales externas en el MS, desde los sensores y cuando está preparada para el enlace de comunicación, una vez aplicado la operación $S k p$. La Figura 9b están las señales externas, provenientes del MS, que son tratadas en la EB, aplicando las operaciones Exp, Rpt. Como sus $T s_{i}$ iguales y $T s_{\text {enlace }}=T s_{0}$, todas las muestras de las señales medidas por los sensores externos son tomadas por el enlace de comunicación y entregadas a la EB. 
Señales MS Entregadas a Tsenlace $=2(20 \mathrm{~min})$

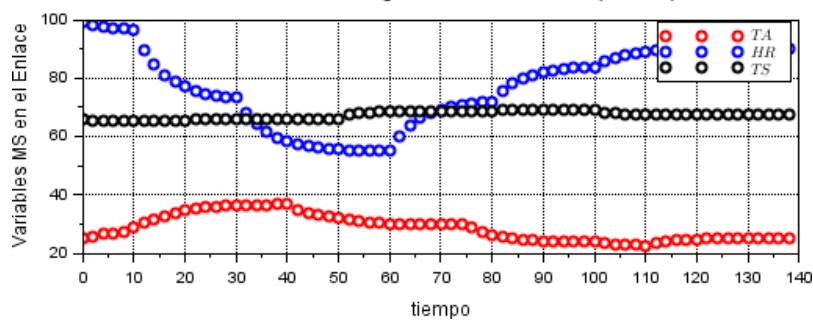

(a)

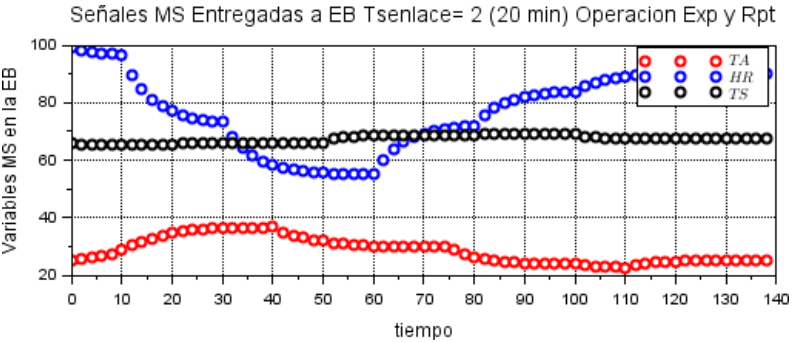

(b)

Figura 9. Salidas de las variables externas del MS con $T s_{i}$ constantes e iguales y $T s_{\text {enlace }}=T s_{0}$. a) En MS con operador $S k p$. b) En la EB con operadores Exp y Rpt.

\subsubsection{Esquemas de Muestreo con $T s_{i}$ constantes, iguales y $T s_{\text {enlace }}>T s_{0}$.}

Los datos a ingresar para la simulación son $T s_{i}=\left[\begin{array}{lll}20 & 20 & 20\end{array}\right]$ min y $T s_{\text {enlace }}=6$ o 60 min. Después de la simulación se generan las señales en 24 horas.

La Figura 10, destaca las salidas de las variables externas entregadas al enlace después de aplicar la operación $S k p$ del MS. En la Figura 11 están las señales externas que son tratadas en la EB, aplicando las operaciones Exp, Rpt e interpolación mediante trazadores. Observando, que al no estar todas las muestras disponibles porque $T s_{\text {enlace }}>T s_{0}$, lo que lleva a que los factores $N s_{i}$ sean iguales a tres (3), arrojando como resultado lo presentado. Por tanto, con Exp inserta muestras en cero y con $R p t$ repite la última muestra ingresada y lleva a una diminución de los tiempos de muestreo.

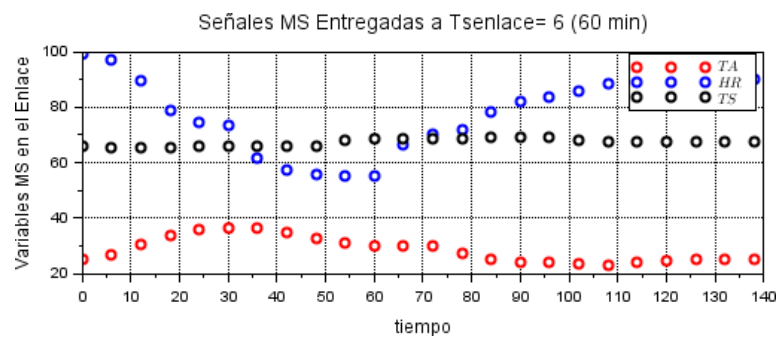

Figura 10. Salidas de las variables externas $S k p$ del MS, $T s_{i}$ constantes e iguales y $T s_{\text {enlace }}>T s_{0}$.

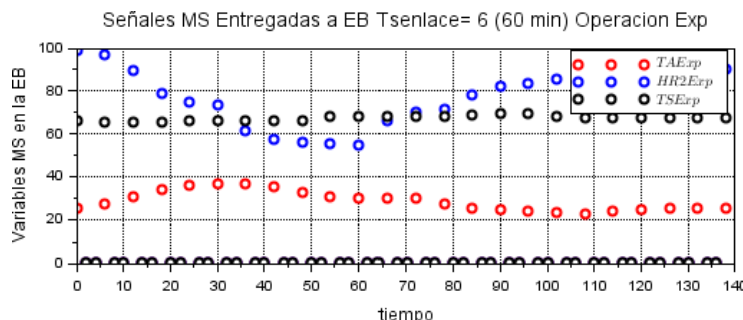

Señales MS Entregadas a EB Tsenlace $=6(60 \mathrm{~min})$ Operacion Rpt

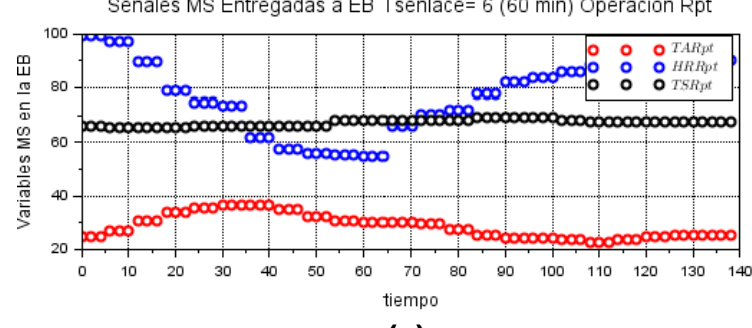

(a)

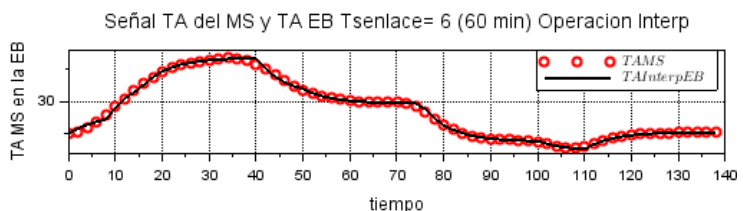

Señal HR del MS y HR EB Tsenlace $=6$ (60 min) Operacion Interp

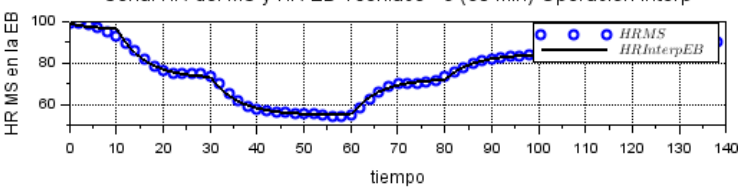

Señal TS del MS y TS EB Tsenlace $=6(60 \mathrm{~min})$ Operacion Interp

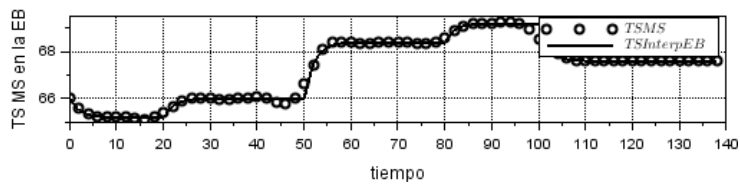

(b) 
Figura 11. Salidas de las variables externas del MS en la EB con $T s_{i}$ constantes e iguales. a) Operador Exp (superior), Operador Rpt (inferior). b) Interpolación caso $T s_{\text {enlace }}>T s_{0}$.

\subsection{Caso Tres: Esquemas de Muestreo con $T s_{i}$ constantes, diferentes y $T s_{\text {enlace }} \geq T s_{0}$.}

En las dos subsecciones siguientes se presentan la discusión y resultados de la simulación cuando $T s_{i}$ son constantes, diferentes y $T s_{\text {enlace }}=T s_{0}$ y $T s_{\text {enlace }}>T s_{0}$. Haciendo una prueba cuando el $T s_{\text {enlace }}$ es variable.

\subsubsection{Esquemas de Muestreo con $T s_{i}$ constantes, diferentes y $T s_{\text {enlace }}=T s_{0}$.}

Para consolidar este caso, se utilizan los datos $T s_{\text {enlace }}=6060$ min y $T s_{i}=\left[\begin{array}{lll}20 & 30 & 20\end{array}\right]$ min. Después de la simulación se generan las señales en 24 horas.

La Figura 12 representa el comportamiento de las señales externas en el MS, desde los sensores y cuando está preparada para el enlace de comunicación, una vez aplicado la operación $S k p$. Como sus $T s_{i}$ diferentes y el $T s_{\text {enlace }}=T s_{0}$, no todas las muestras de las señales medidas por los sensores externos son tomadas por el enlace de comunicación. El número de muestras por señal está determinada por $N s_{i}$. El número de muestras no consideradas por el enlace para TA, HR y TS son dos (2), una (1) y dos (2) y en este caso se saben cuáles son, porque el $S k p$ prepara la primera muestra contenida en el $T S_{\text {enlace }}$. Lo que trae ventajas al querer reproducir estos datos ausentes.

En las Figura 13 están las señales externas, provenientes del MS, que son tratadas en la EB aplicando las operaciones Exp, Rpt e interpolación mediante trazadores. Observando, que las muestras disponibles ocurren cada $T s_{\text {enlace }}$, al necesitar alimentar algún procedimiento que necesite llevarlas a los $T s_{i}$, es cuando los operadores entran en función. El Exp aumenta el periodo de muestreo a $T s_{i}$ haciendo las muestras ausentes igual a cero, por el contrario, el $R p t$ repetirá la última muestra que llega del enlace. Lo que hace atractivo incorporar estos operadores.
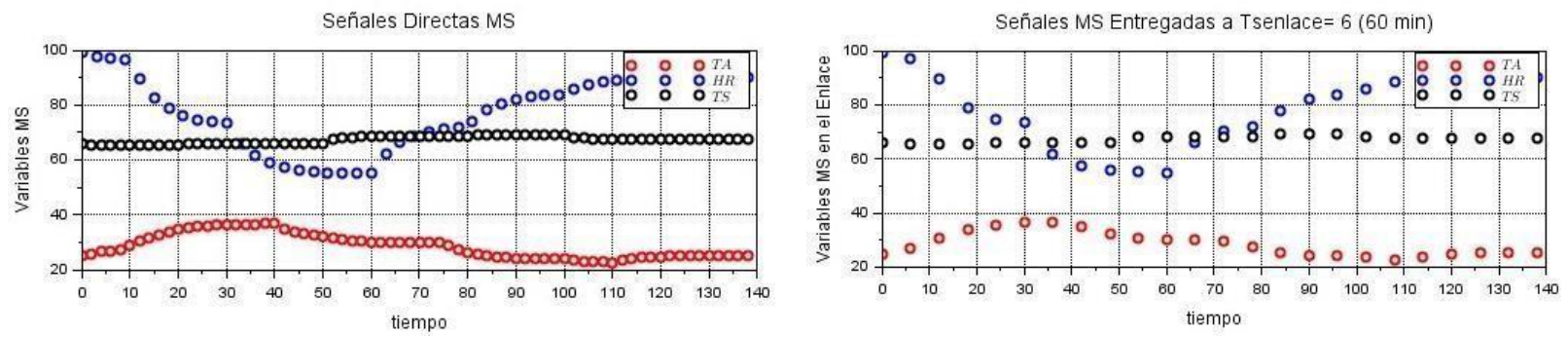

Figura 12. Salidas de las variables externas del MS con $T s_{i}$ constantes, diferentes y $T s_{\text {enlace }}=T s_{0}$. TA, HR y TS (superior). Operador Skp TA, HR y TS (inferior). 


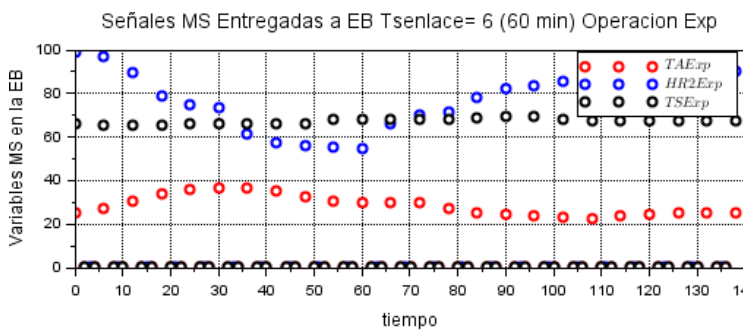

Señales MS Entregadas a EB Tsenlace $=6$ (60 min) Operacion Rpt

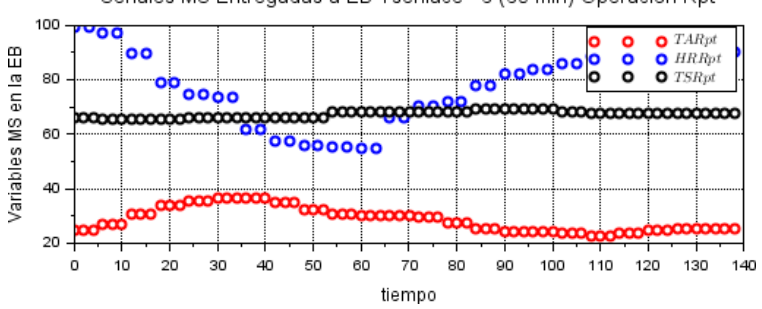

(a)

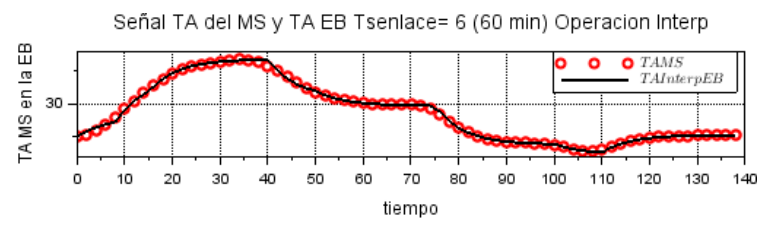

Señal HR del MS y HR EB Tsenlace $=6$ ( 60 min) Operacion Interp

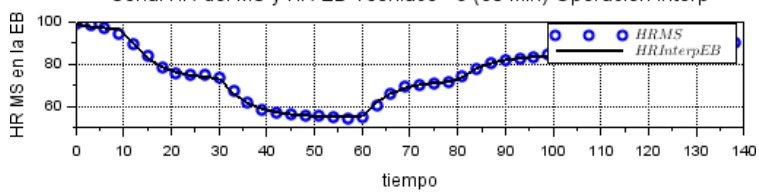

Señal TS del MS y TS EB Tsenlace $=6$ (60 min) Operacion Interp

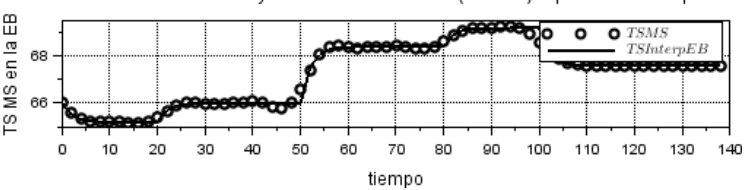

(b)

Figura 13. Salidas de las variables externas del MS en la EB. a) Operador Exp (superior) y Operador $R p t$ (inferior). b) Interpolación caso $T s_{\text {enlace }}=T s_{0}$.

\subsubsection{Esquemas de Muestreo con $T s_{i}$ constantes, diferentes y $T s_{\text {enlace }}>T s_{0}$.}

En este caso, se utilizan los datos $T s_{\text {enlace }}=120120 \mathrm{~min}$, pudiendo ser otro. Con $T s_{i}=$ $\left[\begin{array}{lll}20 & 30 & 20\end{array}\right] \min , T c s_{r}=\left[\begin{array}{ll}20 & 20\end{array}\right] \min$. Después de la simulación se generan las señales en 24 horas.

La Figura 14 representa el comportamiento de las señales externas en el MS, desde los sensores y cuando está preparada para el enlace de comunicación, una vez aplicado la operación $S k p$. Como sus $T s_{i}$ son diferentes y el $T s_{\text {enlace }}>T s_{0}$, no todas las muestras de las señales medidas por los sensores externos son tomadas por el enlace de comunicación.

En las Figura 15 están las señales externas, provenientes del MS, que son tratadas en la EB, aplicando las operaciones Exp, Rpt e interpolación mediante trazadores. Observando, que al no estar todas las muestras disponibles porque el enlace impone $T s_{\text {enlace }}>T s_{0}$, lo que lleva a que los factores $N s_{i}$ sean diferentes, arrojando como resultado lo presentado. Por tanto, Exp inserta muestras en cero y el Rpt repite la última muestra ingresada y llevar a un aumento de los respectivos tiempos de muestreo, lo que es conveniente si es necesario, ingresar dichos datos en controladores a un muestreo dado.

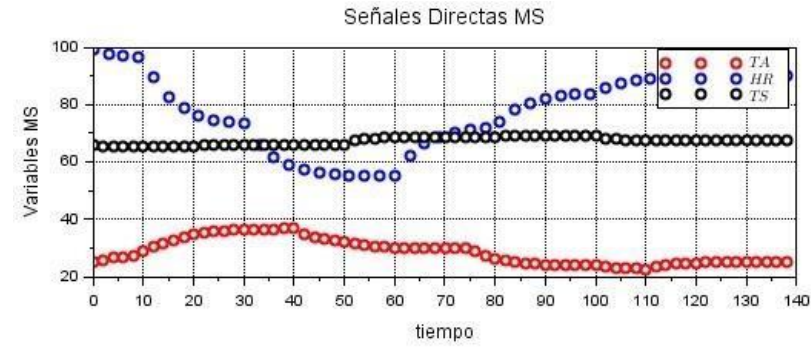

(a)

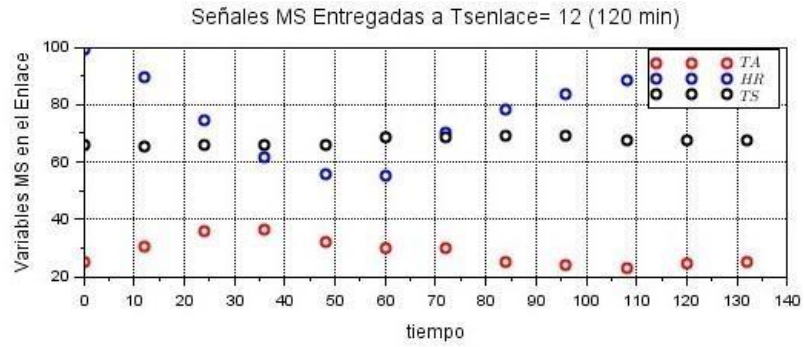

(b)

Figura 14. Salidas de las variables externas del MS con $T s_{i}$ constantes, diferentes y $T s_{\text {enlace }}>T s_{0}$. a) TA, HR y TS. b) Operador $S k p$ TA, HR y TS. 
Señales MS Entregadas a EB Tsenlace $=12(120 \mathrm{~min})$ Operacion Exp

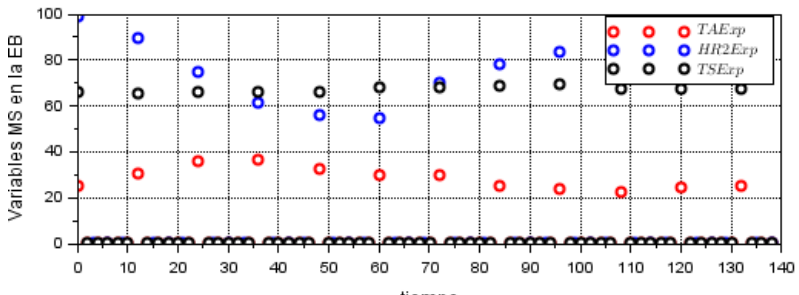

Señales MS Entregadas a EB Tsenlace= $12(120 \mathrm{~min})$ Operacion Rpt

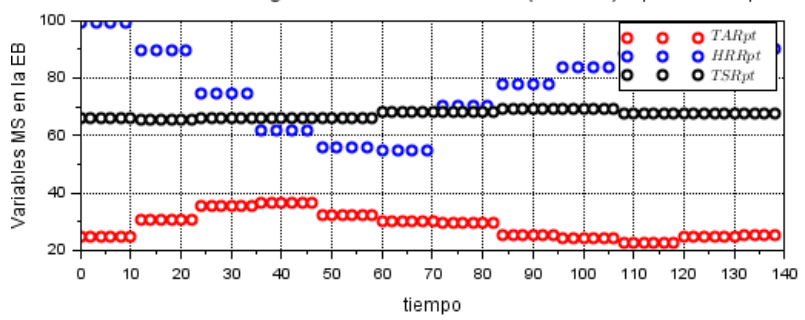

(a)

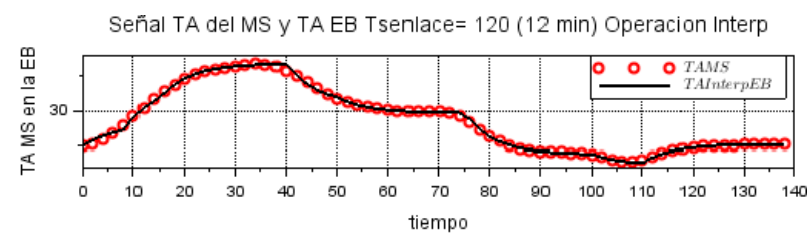

Señal HR del MS y HR EB Tsenlace= 120 (12 min) Operacion Interp

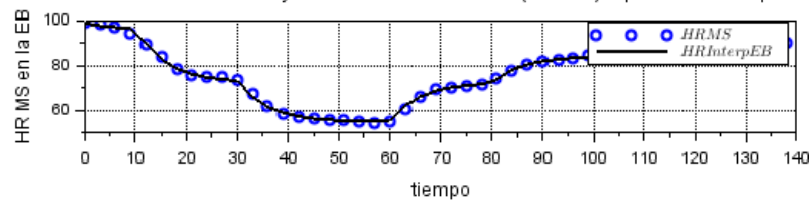

Señal TS del MS y TS EB Tsenlace $=120$ (12 min) Operacion Interp

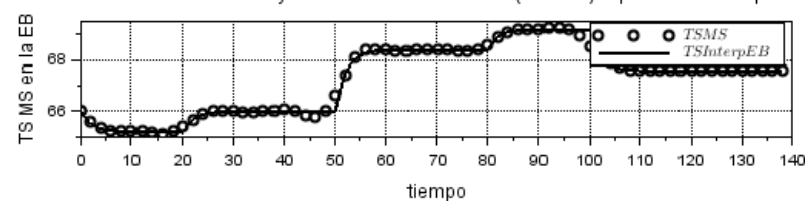

(b)

Figura 15. Salidas de las variables externas del MS en la EB con $T s_{i}$ constantes, diferentes y $T s_{\text {enlace }}>$ $T s_{0}$. A) Operador Exp (superior), Operador Rpt (inferior). b) Interpolación

\subsection{Caso Cuatro: Esquemas de Muestreo con $T s_{i}$ constantes y diferentes con retardos en espera de la activación del enlace.}

Los casos anteriores permiten el análisis de un SMs al variar parámetros dentro de los sistemas MF, haciendo énfasis en las muestras medidas por los respectivos sensores (externos e internos) llevándolos al límite temporal regido por el $T s_{\text {enlace }}$, es decir el plazo de finalización para la preparación de los datos que serán entregados al enlace de comunicación.

No obstante, existen otros en los cuales dichas muestras deben esperar ser atendidas por el enlace ocasionando retardos $\left(\Delta s_{\text {enlace }}\right)$. Debido a las esperas que se presentan al preparar $E_{z}$, antes de que la política de arbitraje del medio compartido señale que recoja el último y lo transmita. Con tal fin, se estableció previamente la condición $T s_{\text {enlace }}>T s_{0}$ para modelar esta situación, donde $E_{z}$ debe estar disponible para el enlace. Por tanto, al introducir el retardo $\Delta s_{\text {enlace }}$ se seguiría recogiendo dichos datos, hasta una eventual sincronización con $T s_{\text {enlace, }}$, asunto que se presentó en la Figura 5 . Vale la pena destacar, que estos retardos también pueden ser ocasionados por otros motivos, siendo su tratamiento similar. Uno de ese motivos son los retardos ocasionados por las interrupciones del procesador al ejecutar la adquisición de las muestras en los procesos.

En la Figura 16 se presenta la ejemplificación del caso a considerar, donde las muestras que están listas y esperan el establecimiento del enlace aparecen enmarcadas en un cuadrado y con un triángulo las que están listas justo en el tiempo límite dado por $T s_{\text {enlace }}$. Observando que en la línea indicada con nueve (9), cuando se activa el $T s_{\text {enlace }}$, dos (2) muestras están esperando y una (1) llega al límite, logrando conformar a $E_{z}$ y ocupan el enlace. Para el siguiente $T S_{\text {enlace }}$, se observa que todas las muestras llegan con el tiempo límite, se preparan en $E_{z}$ y ocupan el enlace. Esto último sucede por la sincronización entre los tiempos de muestreo y $T s_{\text {enlace. }}$ También hay muestras que no son atendidas por el enlace produciendo lo que se llama, datos ausentes. 


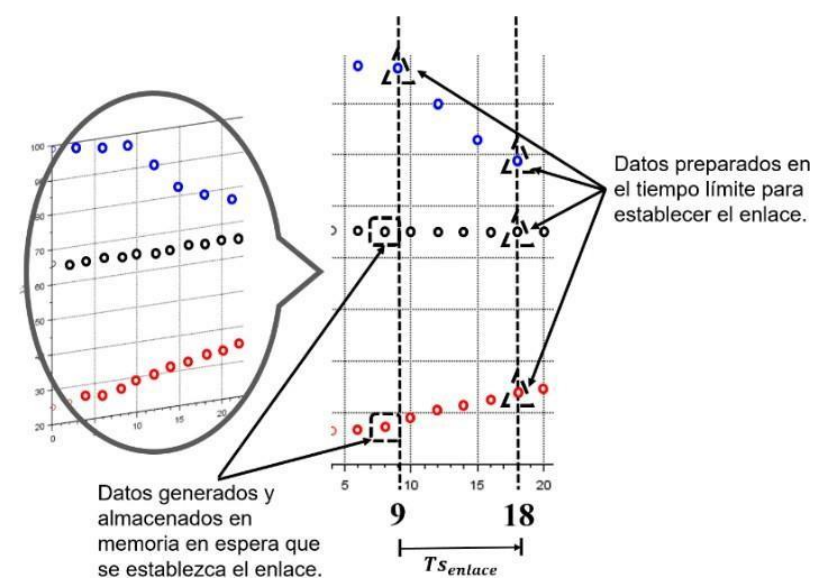

Figura 16. Salidas de las variables del MS con $T s_{i}$ constantes, diferentes y $T s_{\text {enlace }}>T s_{0}$ ampliada para enfatizar las muestras generadas, almacenadas y las que están listas cuando se estable el enlace.

Para la simulación se utilizan los datos $T s_{\text {enlace }}=9$ o $90 \mathrm{~min}$ para forzar los $\Delta s_{\text {enlace, }}$, pudiendo ser otro, $\operatorname{con} T s_{i}=\left[\begin{array}{lll}20 & 30 & 20\end{array}\right]$ min. Después de la simulación se generan las señales en 24 horas.

La Figura 17 representa el comportamiento de las señales externas en el MS, desde los sensores y cuando está preparada para el enlace de comunicación, una vez aplicado la operación $S k p$. Como sus $T s_{i}$ diferentes y el $T s_{\text {enlace }}>T s_{0}$. En esta oportunidad se hace con más detalle, debido a que se desea resaltar lo que sucede con las muestras de TA y TS cuando presentan el $\Delta s_{\text {enlace }}$ y $H R$ se alinea cuando se activa $T S_{\text {enlace. }}$. También se hace una proyección a la gráfica en la parte superior donde se resalta muestras de TA y TS que están alcanzando a $T s_{\text {enlace }}$ pero no son consideradas en la transmisión. Esto es debido a los parámetros MF utilizados en la preparación de $E_{z}$, como condición para la comunicación. Por supuesto no todas las muestras de las señales medidas por los sensores externos son tomadas por el enlace de comunicación.

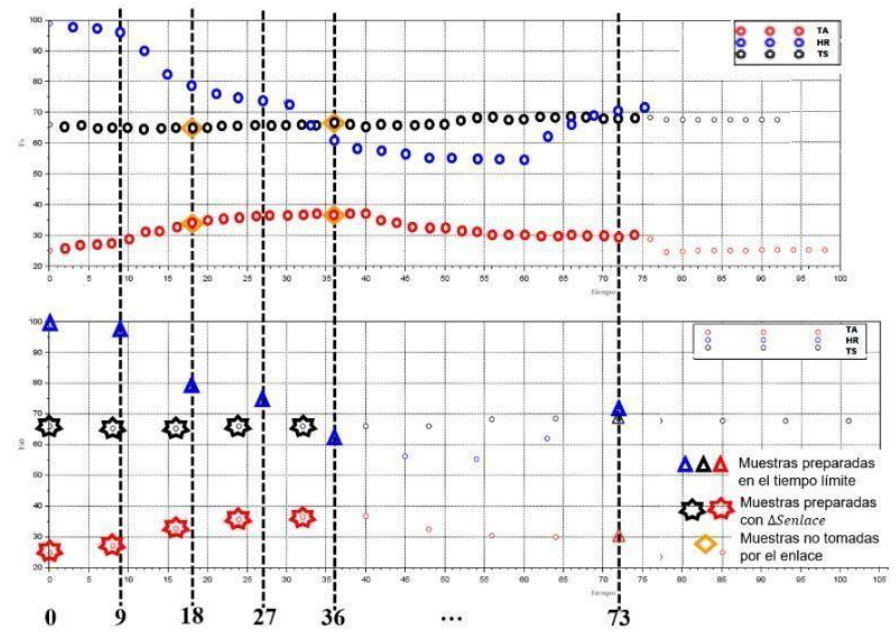

Figura 17. Salidas de las variables externas del MS con $T s_{i}$ constantes, diferentes y $T s_{\text {enlace }}>T s_{0}$ en detalle. TA, HR y TS (superior). Operador $S k p$ TA, HR y TS (inferior).

En las Figura 18 están las señales externas, provenientes del MS, tratadas en la EB con Exp, Rpt e interpolación mediante trazadores. Observando que al no estar todas las muestras disponibles porque el enlace impone $T s_{\text {enlace }}>T s_{0}$, lleva a que los factores $N s_{i}$ sean diferentes. La importancia de tener información del número de muestras originales de cada señal y el momento cuando ocurren, incluyendo 
las ausentes, dan una ventaja a los operadores Exp, Rpt e interpolador mediante trazadores cúbicos, poder generar las respectivas señales en la EB.

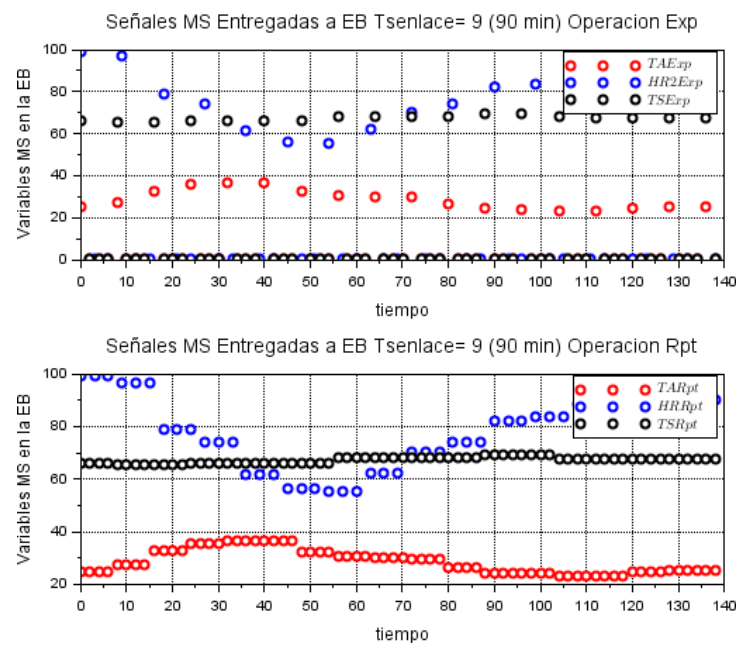

(a)

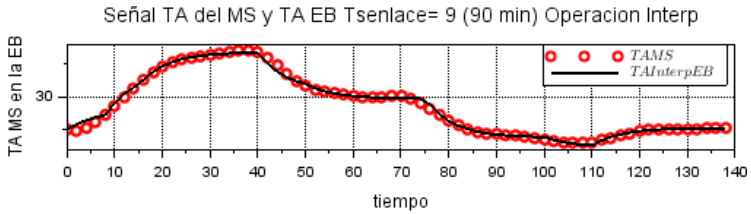

Señal HR del MS y HR EB Tsenlace $=9(90 \mathrm{~min})$ Operacion Interp

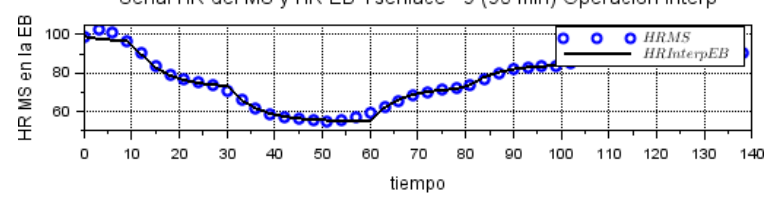

Señal TS del MS y TS EB Tsenlace $=9(90 \mathrm{~min})$ Operacion Interp

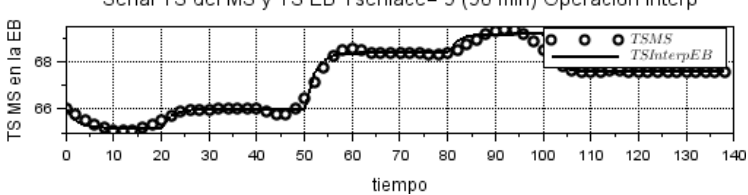

(b)

Figura 18. Salidas de las variables externas del MS en la EB con $T s_{i}$ constantes y diferentes, $T s_{\text {enlace }}>$ $T s_{0}$. a) Operador Exp y Rpt. b) Interpolación mediante trazadores.

\section{CONCLUSIONES}

Se desarrolla un modelo de sistema multisensor considerando múltiples señales con periodos de muestreo que pueden ser diferentes del proceso y datos de estado, incluyendo la limitación temporal del enlace de comunicación. El sistema consta de los componentes: multisensor, enlace de comunicación y estación base. Además, se desarrolló un modelo de simulación en Scilab/Xcos para ejercitar diferentes escenarios.

El modelo definido bajo los lineamientos del muestreo no convencional permite analizar la influencia que tienen los diferentes tiempos de muestreo de las múltiples señales con periodos de muestreo que pueden ser diferentes del proceso y datos de configuración tratadas y generadas por el multisensor, como sucede en la realidad, ya que se establecen esquemas de muestreo que propician contabilizar el número de muestras que recoge el multisensor en un periodo global. También se contribuye al estudio del enlace de comunicación que impone una restricción temporal $T s_{\text {enlace }}$ respecto a la limitación en las frecuencias de muestreo de las señales transmitidas y recibidas.

El modelo ofrece un perfil temporal de las secuencias de datos sensoriales bajo diferentes esquemas de muestreo, permitiendo presentar la evolución de las señales con pérdidas o ausencias de muestras, las cuales al ser tratadas sistemáticamente con operadores multifrecuencia y funciones de interpolación para su reconstrucción, mitigan ciertas ausencias de datos y conducen a resultados utilizables para la abstracción del mundo real.

El modelo permite definir las especificaciones funcionales de diseño en la descripción del sistema computacional empotrado guiado por la arquitectura de un sistema multisensor. Así como también, colaborar con la misión de proveer mecanismos, que en forma temprana, sean capaces de presentar escenarios para analizar y tomar decisiones.

Finalmente, la efectividad del modelo se ilustra mediante simulaciones numéricas.

\section{AGRADECIMIENTOS}




\section{REFERENCIAS}

Akyildiz, I. F. y Kasimoglu, I. H. (2004). Wireless Sensor and Actor Networks: Research Challenges. Ad Hoc Networks Journal, 2(4), 351-367. https://doi.org/10.1016/i.adhoc.2004.04.003

Albertos, P. y Salt, J. (2011). Non-Uniform Sampled-Data Control of Mimo Systems. Annual Reviews in Control, 35(1), 65-76. https://doi.org/10.1016/i.arcontrol.2011.03.004

Aranda-Escolástico, E., Salt, J., Guinaldo, M., Chacón, J. y Dormido, S. (2018). Optimal Control for Aperiodic Dual-Rate Systems With Time-Varying Delays. Sensors (Basel, Switzerland), 18(5), 1 19. Article 1491. https://dx.doi.org/10.3390/s18051491

Bamieh, B. y Pearson, J.B. (1991). A lifting technique for linear periodic systems with applications to sampled-data control. Systems \& Control Letters, 17(2), 79-88. https://doi.org/10.1016/0167$\underline{6911(91) 90033-B}$

Chapra, S. y Canale, R. (2015). Métodos Numéricos para Ingenieros (7ª ed.). Mcgraw-Hill/Interamericana Editores, S.A. de C.V.

Cimino, M. y Pagilla, P. R. (2010). Design of linear time-invariant controllers for multirate systems. Automatica, 46, 1315-1319.

Coffey, T. C. y Williams, I. J. (1966). Stability Analysis of Multiloop, Multirate Sampled Systems. Journal of Guidance, Control, and Dynamics, 4(12), 2178-2190. https://doi.org/10.2514/3.3874

Crochiere, R. E. y Rabiner, L. R. (1981). Interpolation and decimation of digital signals-A tutorial review. Proceedings of the IEEE, 69(3), 300-331. https://doi.org/10.1109/PROC.1981.11969

Deb Majumder, B., Roy, J. K. y Padhee, S. (2019). Recent Advances in Multifunctional Sensing Technology on a Perspective of Multi-Sensor System: A Review. IEEE Sensors Journal, 19(4), 1204-1214. https://doi.org/10.1109/JSEN.2018.2882239

Ding, F., Quu, L. y Chen, T. (2009). Reconstruction of continuous-time systems from their non-uniformly sampled discrete-time systems. Automatica, 45(2), $324-332$. https://doi.org/10.1016/i.automatica.2008.08.007

Dorf, R. C. y Bishop, R. H. (2016). Modern Control Systems (13 th ed.). Pearson Education Limited.

Ghosal, M. y Rao, V. (2019). Fusion of Multirate Measurements for Nonlinear Dynamic State Estimation of the Power Systems. IEEE Transactions on Smart Grid, 10(1), 216-226. https://doi.org/10.1109/TSG.2017.2737359

Glasson, D. P. (1983). Development and Applications of Multirate Digital Control. IEEE Control Systems Magazine, 3(4), 2-8. https://doi.org/10.1109/MCS.1983.1104772

Godbout, L. F., Jordan, D. y Apostolakis, I. S. (1990) Closed-loop model for general multirate digital control systems. IEE Proceedings D - Control Theory and Applications, 137(5), 329-336. https://doi.org/10.1049/ip-d.1990.0043

Goyal, R. y Dhingra, M. (2019). Programming in Scilab. Alpha Science.

Ha, S. y Teich, J. (Ed). (2017). Handbook of Hardware/Software Codesign. Springer Science+Business Media. https://doi.org/10.1007/978-94-017-7267-9 
ISO/IEC/IEEE. (2010a). ISO/IEC/IEEE 21451-1: 2010. Information technology -- Smart transducer interface for sensors and actuators -- Part 1: Network Capable Application Processor (NCAP) information model. http://doi.org/10.1109/IEEESTD.2010.5668469

ISO/IEC/IEEE. (2010b). ISO/IEC/IEEE 21450: 2010. Information technology -- Smart transducer interface for sensors and actuators. Common functions, communication protocols, and Transducer Electronic Data Sheet (TEDS) formats. https://doi.org/10.1109/IEEESTD.2010.5668466

Jia, X., Ma, W., Yang, F. y Zhang, D. (2017). Matching mechanism for networked control systems with multirate sampling. Institution of Engineering \& Technology Control Theory \& Applications, 11(16), 2848-2857. https://doi.org/10.1049/iet-cta.2017.0039

Kakarla J., Majhi, B. y Battula, R. B. (2015). "Comparative Analysis of Routing Protocols in Wireless Sensor-Actor Networks: A Review". International Journal of Wireless Information Networks, 22, 220-239. https://doi.org/10.1007/s10776-015-0271-2

Kordestani, M., Dehghani, M., Moshiri, B. y Saif, M. (2020). A New Fusion Estimation Method for MultiRate Multi-Sensor Systems With Missing Measurements. IEEE Access, 8, 47522-47532. https://doi.org/10.1109/ACCESS.2020.2979222

Kranc, G. (1957). Input-output Analysis of Multirate Feedback Systems. Institute of Radio Engineers Transactions Automatic Control, 3(1), 21-28. https://doi.org/10.1109/TAC.1957.1104783

Laszlo, C. y Zhexembayeva, N. (2011). Embedded Sustainability. The Next Big Competitive Advantage. Stanford University Press. https://doi.org/10.4324/9781351278324

Lathi, B. P. y Green, R. A. (2019). Essentials of Digital Signal Processing. Cambridge University Press.

Liu, Y., Peng, Y., Wang, B. L., Yao, S. R. y Liu, Z. H. (2017). Review on Cyber-physical Systems. IEEE/CAA Journal of Automatica Sinica, 4(1), 27-40. https://doi.org/10.1109/JAS.2017.7510349

Meinsma, G. y Mirkin, L. (2010a). Sampling from a system-theoretic viewpoint: part I concepts and tools. IEEE Transactions on Signal Processing, 58(7), 3578-359. https://doi.org/10.1109/TSP.2010.2047641

Meinsma, G. y Mirkin, L. (2010b). Sampling from a system-theoretic viewpoint: Part II - Noncausal solutions. IEEE transactions on signal processing, 58(7), 3591-3606.

https://doi.org/10.1109/TSP.2010.2047642

Muangprathuba, J., Boonnam, N., Kajornkasirata, S., Lekbangpong, N., Wanichsombata, A. y Nillaor, P. (2019). IoT and Agriculture Data Analysis for Smart Farm. Computers and Electronics in Agriculture, 156, 467-474. https://doi.org/10.1016/..compag.2018.12.011

Nwe, M. S. y Tun, H. M. (2017). Implementation of Multisensor Data Fusion Algorithm. International Journal of Sensors and Sensor Networks, 5(4), 48-53. https://doi.org/10.11648/j.ijssn.20170504.11

Oppenheim, A. V., Willsky, A. S. y Hamid Nawab, S. (2013). Signals and Systems: Pearson New International Edition (2nd ed.). Pearson Education Limited.

Pradilla J., Esteve, M. y Palau, C. (2018). SOSFul: Sensor Observation Service (SOS) for Internet of Things (IoT). IEEE Latin American Transactions, 16(4), 1276-1283. https://doi.org/10.1109/TLA.2018.8362168 
Roehm, H., Oehlerking, J., Woehrle, M. y Althoff, M. (2019). Model Conformance for Cyber-physical Systems: A Survey. ACM Transactions on Cyber-Physical Systems, 3(3), Article 30, 26p. https://doi.org/10.1145/3306157

Salt J. y Albertos P. (2005). Model-based Multirate Controllers Desing. IEEE Transactions on Control Systems Tecnology, 13(6), 988-997. https://doi.org/10.1109/TCST.2005.857410

Salt, J., Casanova, V., Cuenca, A. y Pizá, R. (2008). Sistemas de Control Basados en Red. Modelado y Diseño de Estructuras de Control. Revista Iberoamericana de Automática e Informática Industrial, 5(3), 5-20. https://polipapers.upv.es/index.php/RIAl/article/view/8290

Salt, J., Cuenca, A., Palau, F. y Dormido, S. (2014). Multirate Control Strategy to the Slow Sensors Problem: An Interactive Simulation Tool for Controller Assisted Design. Sensors, 3, 4086-4110. https://doi.org/10.3390/s140304086

Scilab. (2020). Scilab (Versión 6.1.0) [Software de Análisis Numérico Computacional, Código Abierto en Línea]. Engineering System International Group. https://www.scilab.org/

Tan, L. y Jiang, J. (2018). Digital Signal Processing: Fundamentals and Applications (3rd ed.). Elsevier Science Publishing.

Teich, J. (2012). Hardware/Software Codesign: The Past, the Present, and Predicting the Future. Proceeding of the IEEE, 100, 1411-1430. https://doi.org/10.1109/JPROC.2011.2182009

Thompson, P. M. (1985). Gain and Phase Margins of Multirate Sampled-Data Feedback Systems. International Journal of Control. 44(3), 833-846. https://doi.org/10.1080/00207178608933635

Yan, L., Li, X. R., Xia Y. y Fu M. (2015). Modeling and estimation of asynchronous multirate multisensor system with unreliable measurements. IEEE Transactions on Aerospace and Electronic Systems, 51(3), 2012-2026. https://doi.org/10.1109/TAES.2015.140462.

Zhang, D., Bai, W. y Jia, X. (2015). Periodic Switched Control of Dual-Rate Sampled-Data Systems. Mathematical Problems in Engineering, 2015, Article ID 515232. http://dx.doi.org/10.1155/2015/515232

Zhu, Q., Lu, K. Zhu, Y. y Xie, G. (2016). Modelling and State Feedback Control of Multi-Rate Networked Control Systems with Both Short Time Delay and Packet Dropout. International Journal of Innovative Computing, Information and Control, 12(3), 779-793. https://doi.org/10.24507/ijicic.12.03.779 


\section{Notas Biográficas}



Alfonso Alfonsi es Doctor en Ciencias, MSc. en Instrumentación ambas de la Universidad Central de Venezuela e Ing. Electricista de la Universidad de Oriente (UDO), Venezuela. Profesor Titular de la UDO e Investigador activo y acreditado en el SAI y PEII. Coordinador del Grupo de Investigación Arquitecturas de Sistemas de Control. Miembro de la Sociedad Venezolana de Computación, IEEE en sus sociedades de: Control Systems, Robotics and Automation, Instrumentation and Measurement. Ha publicado artículos en revistas científicas. El Dr. Alfonsi dirige su investigación a los sistemas empotrados con requisitos heterogéneos y complejos para del desarrollo sustentable.

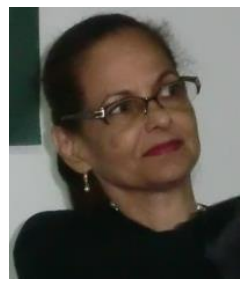

Raiza Yánez es Doctora en Gerencia de la Universidad de Yacambú, MSc. en Cs. Administrativas mención Gerencia General e Ing. Industrial de la Universidad de Oriente (UDO), Venezuela. Profesora Titular de la UDO e Investigadora activa y acreditada en el SAI y PEIl, miembro del Grupo de Investigación Arquitecturas de Sistemas de Control. Coordina el Programa de Actualización Profesional Sistemas de Gestión de la Calidad. Ha publicado artículos en revistas científicas. La Dra. Yánez dirige su investigación al pensamiento complejo y desarrollo sustentable.

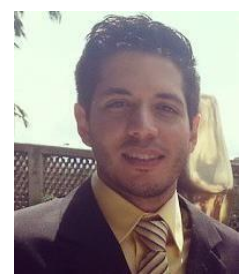

Alfonso R. Alfonsi es Ingeniero Electricista de la Universidad de Oriente, Barcelona, Venezuela, Mención Honorífica Magna Cum Laude. Investigador asociado. Miembro del Grupo de Investigación Arquitecturas de Sistemas de Control. Más de ocho años de experiencia en la industria petrolera internacional en el área de operaciones de perforación direccional de pozos. Especialista en herramientas de medición y registro mientras se perfora (MWD y LWD, por su siglas en inglés), desde su ensamblaje, programación y operación en campo; solución de problemas, asistencia técnica y análisis de fallas.

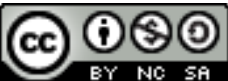

Esta obra está bajo una licencia de Creative Commons Reconocimiento-NoComercial-Compartirlgual 2.5 México. 